TITLE:

\title{
Crystal-plasticity finite-element analysis of inelastic behavior during unloading in a magnesium alloy sheet
}

\section{$\operatorname{AUTHOR}(\mathrm{S})$ :}

Hama, Takayuki; Takuda, Hirohiko

\section{CITATION:}

Hama, Takayuki ...[et al]. Crystal-plasticity finite-element analysis of inelastic behavior during unloading in a magnesium alloy sheet. International Journal of Plasticity 2010, 27(7): 1072-1092

ISSUE DATE:

2010-12-04

URL:

http://hdl.handle.net/2433/141817

\section{RIGHT:}

(C) 2010 Elsevier Ltd.; This is not the published version. Please cite only the published version.; この論文は出版社版でありません。引用の際に は出版社版をご確認ご利用ください。 
Crystal-Plasticity Finite-Element Analysis of Inelastic Behavior During Unloading in a Magnesium Alloy Sheet

Takayuki HAMA ${ }^{* 1, * 2}$

Hirohiko TAKUDA*1

*1 Graduate School of Energy Science, Kyoto University, Yoshida-honmachi, Sakyo-ku, Kyoto 606-8501, Japan

*2 VCAD System Research Program, RIKEN, 2-1 Hirosawa, Wako-shi, Saitama, 351-0198, Japan

Corresponding author: Takayuki HAMA

Graduate School of Energy Science, Kyoto University

Yoshida-Honmachi, Sakyo-ku, Kyoto 606-8501, Japan

TEL: $\quad+81-(0) 75-753-5418$

FAX: $\quad+81-(0) 75-753-5428$

E-mail: hama@energy.kyoto-u.ac.jp 


\section{ABSTRACT}

A crystal-plasticity finite-element analysis of the loading-unloading process under uniaxial tension of a rolled magnesium alloy sheet was carried out, and the mechanism of the inelastic response during unloading was examined, focusing on the effects of basal and nonbasal slip systems. The prismatic and basal slip systems were mainly activated during loading, but the activation of the prismatic slip systems was more dominant. Thus the overall stress level during loading was determined primarily by the prismatic slip systems. The prismatic slip systems were hardly activated during unloading because the stress level was of course lower than that during loading. On the other hand, because the strength of the basal slip systems was much lower than that of the prismatic slip systems, the basal slip systems would be easily activated under the stress level during unloading in the opposite direction when their Schmid's resolved shear stresses changed signs because of the inhomogeneity of the material. These results indicated that one explanation for the inelastic behavior during unloading was that the basal slip systems were primarily activated owing to their low strengths compared to that of the prismatic slip systems. Numerical tests using the sheets with random orientations and with the more pronounced texture were conducted to further examine the mechanism.

Keywords:

Magnesium

B. Crystal plasticity

C. Finite elements

B. Cyclic loading

B. Polycrystalline material 


\section{Introduction}

Magnesium (Mg) alloys are the lightest materials used for structural components, and their specific strength and stiffness are high (Mordike and Ebert, 2001). Moreover, their functional properties such as recyclability (Mordike and Ebert, 2001; Chino et al., 2006a) and electromagnetic shielding (Mordike and Ebert, 2001; Chino et al., 2006a; Kim et al., 2008) are also superior. Mg alloys have recently received attention because of the increasing demand for lightweight materials for automobile and electrical devices (Mordike and Ebert, 2001) to reduce their environmental impact.

Conventionally, applications made of $\mathrm{Mg}$ alloys are manufactured by die casting and thixoforming (Kim et al., 2008; Kaneko and Sugamata, 2004; Lee et al., 2007). The press forming of Mg alloy sheets has recently attracted attention because it can further expand the use of Mg alloys for structural components. Many studies on the press forming of $\mathrm{Mg}$ alloy sheets (Kim et al., 2008; Kaneko and Sugamata, 2004; Lee et al., 2007; Doege and Droder, 2001; Chen et al., 2003; Lee et al., 2002; Chen and Huang, 2003; Bruni et al., 2006; Hama et al., 2010a) have been carried out, and various components are manufactured by press forming, such as the housing of laptop computers and cellular phones, and automobile body structures.

To increase the number of applications of press forming, it is essential to understand the mechanical properties of $\mathrm{Mg}$ alloy sheets. They are significantly different from those of conventional structural sheet metals such as steel and aluminum alloys because of the hexagonal close-packed (hcp) structure. For instance, the stress-strain curves obtained by uniaxial tensile and compressive tests of rolled Mg alloy sheets at room temperature are notably different (Lou et al., 2007). This strong asymmetric behavior arises from the direction-dependent $\left\{\begin{array}{llll}1 & 0 & \overline{1} & 2\end{array}\right\}$ twinning, because a strong basal texture with most of the $c$-axes aligned in the sheet normal 
direction is initially formed in the rolled Mg alloy sheets (Lou et al., 2007; Chino et al., 2006b). The strong basal texture also yields poor stretch formability of $\mathrm{Mg}$ alloy sheets regardless of temperature because of the small through-thickness strain (Chino et al., 2008a; Koike, 2005).

Another characteristic behavior of $\mathrm{Mg}$ alloy sheets is a strong inelastic response during unloading, which is the main topic of this study. Figure 1 shows the stress-strain curves obtained from uniaxial tensile tests of Mg alloy, mild steel, and aluminum sheets. Clearly the Mg alloy sheet exhibits a strong inelastic deformation during unloading, and the gradient during unloading is apparently smaller than Young's modulus E. Such inelastic behavior during unloading can also be observed in other metals such as uranium (Jones and Munro, 1953), zirconium (Reed-Hill et al., 1965), and steels (Pérez, et al., 2005; Cleveland and Ghosh, 2002; Luo and Ghosh, 2003). Cáceres et al. (2003) and Mann et al. (2007) first examined this behavior in detail in a cast Mg alloy and reported that the inelastic strain increases up to a plastic strain of about $1-2 \%$ and slightly decreases at larger strains. More recently, Muránsky et al. (2009) carried out a cyclic

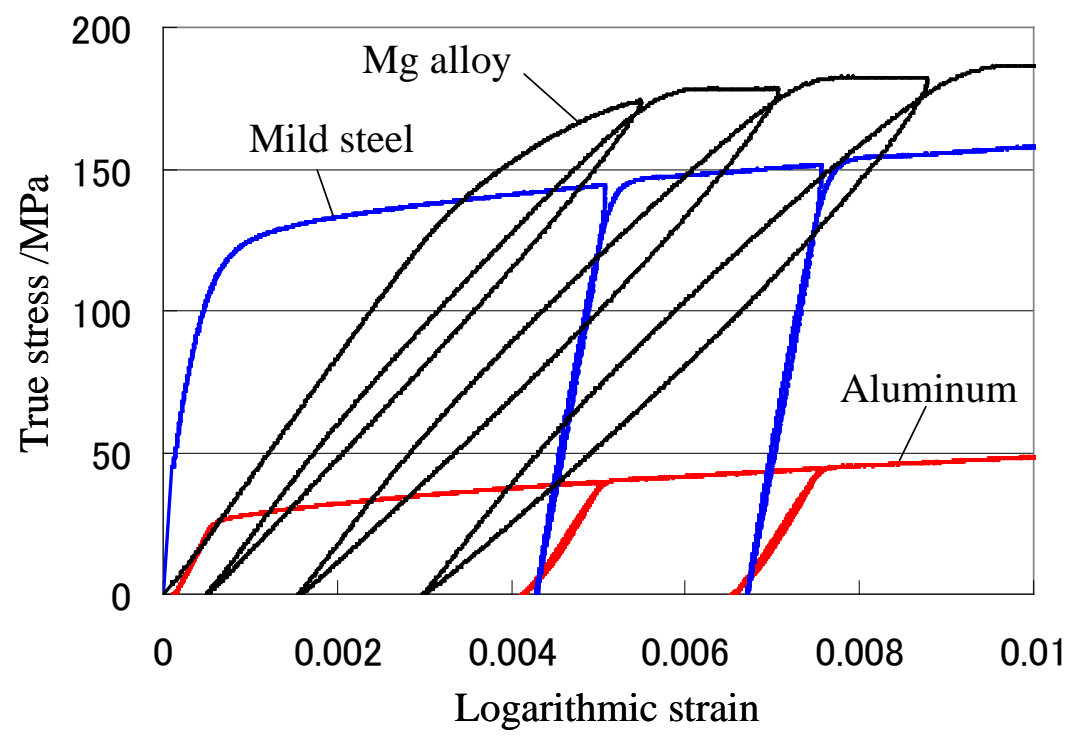

Fig. 1 Inelastic behavior during unloading in Mg alloy, mild steel, and aluminum sheets. 
loading-unloading test of an extruded AZ31 Mg alloy and showed that its inelastic response during unloading is more pronounced under compression than tension. Li and Enoki (2008) reported similar behavior in pure $\mathrm{Mg}$.

Generally it is understood that such inelastic behavior during unloading in metals has several origins such as the presence of mobile dislocations (Reed-Hill et al., 1965; Pérez, et al., 2005; Cleveland and Ghosh, 2002), twinning (Jones and Munro, 1953; Reed-Hill et al., 1965; Cáceres et al., 2003; Mann et al., 2007), and stress-induced phase transformations (Goo et al., 1985). Researchers have experimentally investigated the cause of this behavior in pure $\mathrm{Mg}$ and $\mathrm{Mg}$ alloys using in situ neutron diffraction (Muránsky et al., 2009), metallographic observation (Mann et al., 2007), and acoustic emission (Li and Enoki, 2008). They have concluded that the observed inelastic behavior can be understood in terms of the partial reversal of $\left\{\begin{array}{llll}1 & 0 & \overline{1} & 2\end{array}\right\}$ twinning, i.e., untwinning, upon unloading. Muránsky et al. (2009) presumed that the untwinning process upon unloading is driven by a peculiar internal redistribution of stresses. Although the role of untwinning in inelastic behavior during unloading has been investigated in previous studies (Cáceres et al., 2003; Mann et al., 2007; Muránsky et al., 2009), the effects of other factors such as basal and nonbasal slip have not been examined in detail. For instance, if the untwinning were to be induced by the peculiar internal redistribution of stresses during unloading, basal slip would also be induced during unloading because of their low strengths. However, the effects of basal slip have not been investigated and are still unclear. Koike (2009) recently explained, based on a simple analysis, that this inelastic behavior cannot be explained solely by the reversal of twinning and that the basal slip systems in the hcp structure should also affect the inelastic behavior. This seems reasonable, but it should be carefully examined either experimentally or theoretically. 
The above survey indicates that the mechanism of the inelastic behavior during unloading is still unclear and is open to discussion. Furthermore, because this behavior plays an important role in determining the amount of springback in press forming, as discussed in many previous studies (e.g., Luo and Ghosh, 2003; Yoshida and Uemori, 2003; Morestin and Boivin, 1996), it is worth investigating.

Crystal-plasticity models are powerful tools for analyzing numerically the interaction between mesoscopic crystalline and macroscopic deformation in metals. Recently, the deformation of hcp metals including Mg alloys have been studied using either finite-element methods (Staroselsky and Anand, 2003; Graff et al., 2007; Prakash et al., 2009; Tang et al., 2009; Mayama et al., 2009), self-consistent models (Choi et al., 2009; Proust et al., 2009; Clausen et al., 2008; Agnew and Duygulu, 2005; Turner and Tomé, 1994; Lebensohn and Tomé, 1993), a Taylor model (Lévesque et al., 2010; Wu et al., 2007; Salem et al., 2005; Styczynski et al., 2004), or a combination of approaches (Walde and Riedel, 2007). These studies showed that the stress-strain response and texture evolution of Mg alloys can be reasonably predicted at least for monotonic deformation. A crystal-plasticity analysis of the unloading process of Mg alloys would also be effective for examining the mechanism of the inelastic behavior, but such a study has not yet to our knowledge been carried out.

This paper is concerned with a crystal-plasticity finite-element analysis of the loadingunloading process of a Mg alloy sheet with a rolling texture. First, the simulation results for the behavior in loading-unloading under uniaxial tension in the rolling direction (RD) were compared with the corresponding experimental results. Subsequently, the mechanism of the inelastic behavior during unloading was examined in detail in terms of macroscopic and 
mesoscopic crystalline deformation, and particular attention was focused on the effect of basal and nonbasal slip.

\section{Crystal-Plasticity Finite-Element Formulation}

\subsection{Virtual Work}

The formulation used in the crystal-plasticity finite-element method code developed in this study is briefly described below. An updated Lagrangian rate formulation is used to describe the finite deformation. The rate form of the equilibrium equations and boundary conditions at time $t$ is equivalently expressed by the principle of virtual velocity in the form

$$
\int_{V} \dot{\boldsymbol{\Pi}}^{\mathrm{T}}:\left(\delta \boldsymbol{v} \otimes \nabla_{\boldsymbol{x}}\right) d V=\int_{S_{1}} \dot{\boldsymbol{f}} \cdot \delta \boldsymbol{v} d S,
$$

where $V$ denotes the domain occupied by the body and $S$ denotes its boundary at time $t . S_{1}$ is the part of the boundary $S$ on which the nominal surface traction $f$ is prescribed. $\delta v$ is the virtual velocity field satisfying the condition $\delta v=0$ on the velocity boundary $S_{2}$. $\Pi$ is the first PiolaKirchhoff stress tensor. The superscript $\mathrm{T}$ denotes the transpose. Assuming that all the rate-form relations are preserved from time $t$ to $t+\Delta t$, where $\Delta t$ is a small time increment, an incremental form of the principle of virtual velocity can be written in the form (Kawka and Makinouchi, 1995; Hama et al., 2008)

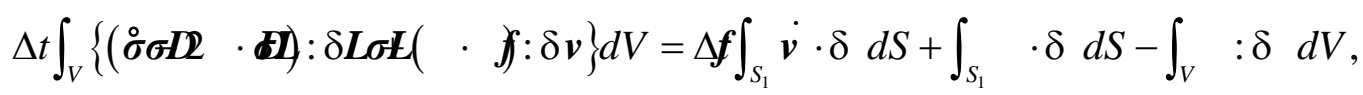

where $\stackrel{\circ}{\sigma}$ is the Jaumann rate of the Cauchy stress tensor, $\boldsymbol{L}$ is the velocity gradient tensor, and $\boldsymbol{D}$ is the strain rate tensor, which is the symmetric part of $\boldsymbol{L}$. The second and third terms of the righthand side remain in order to cancel the nonequilibrated forces arising from the explicit time- 
marching algorithm. To prevent an excessive increase of the nonequilibrium between external and internal forces, the generalized $r_{\min }$-strategy is employed to limit the size of the increment.

\subsection{Constitutive Formulation for Rate-Dependent Crystal-Plasticity Model}

The crystal-plasticity model employed in this study follows the formulations developed in the literature (Graff et al., 2007; Mayama et al., 2009; Pierce et al., 1983; Asaro and Needleman, 1985). We assume that the velocity gradient $\boldsymbol{L}$ consists of elastic and plastic parts, respectively denoted $\boldsymbol{L}^{\mathrm{e}}$ and $\boldsymbol{L}^{\mathrm{p}}$, in the form

$$
\boldsymbol{L}=\boldsymbol{L}^{\mathrm{e}}+\boldsymbol{L}^{\mathrm{p}}
$$

The plastic velocity gradient $\boldsymbol{L}^{\mathrm{p}}$ is related to the slip rate $\dot{\gamma}^{(\alpha)}$ of the $\alpha$ slip system in the form

$$
\boldsymbol{L}^{\mathrm{p}}=\sum_{\alpha=1}^{N} \dot{\gamma}^{(\alpha)}\left(\boldsymbol{s}^{(\alpha)} \otimes \boldsymbol{m}^{(\alpha)}\right)
$$

where the unit vectors $\boldsymbol{s}^{(\alpha)}$ and $\boldsymbol{m}^{(\alpha)}$ are the slip direction and the slip plane normal, respectively, and $N$ is the number of slip systems in a grain. Decomposing Eq. (4) into symmetric and asymmetric parts yields the plastic strain rate $\boldsymbol{D}^{\mathrm{p}}$ and the plastic spin $\boldsymbol{W}^{\mathrm{p}}$ as

$$
\begin{aligned}
& \boldsymbol{D}^{\mathrm{p}}=\sum_{\alpha=1}^{N} \dot{\gamma}^{(\alpha)} \boldsymbol{p}^{(\alpha)}, \text { with } \boldsymbol{p}^{(\alpha)}=\frac{1}{2}\left(\boldsymbol{s}^{(\alpha)} \otimes \boldsymbol{m}^{(\alpha)}+\boldsymbol{m}^{(\alpha)} \otimes \boldsymbol{s}^{(\alpha)}\right), \\
& \left.\mathbf{W} \boldsymbol{\delta}=\sum_{\alpha=1}^{N} \dot{\gamma}^{(\alpha)}{ }^{(\alpha)} \text {, with } \boldsymbol{\omega s}^{(\alpha)}=\frac{1}{2} \boldsymbol{n}^{(\alpha)} \otimes \boldsymbol{m}^{(\alpha)}-\boldsymbol{s}^{(\alpha)} \otimes(\alpha)\right) .
\end{aligned}
$$

The stress is assumed to be determined by the elastic strain rate $\boldsymbol{D}^{\mathrm{e}}$, thus the elastic constitutive equations can be given as

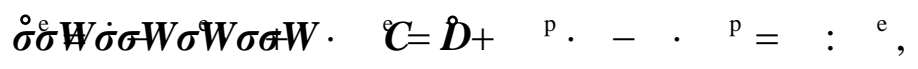

where $\boldsymbol{C}$ is the elastic constitutive moduli. Introducing Eq. (5) into the third equation in (7) gives 


$$
\stackrel{\circ}{\boldsymbol{C}}=\mathbf{D}: \quad-\left(\sum_{\alpha=1}^{N} \mathbb{C}^{(\alpha)} \boldsymbol{p}: \quad(\alpha)\right)
$$

Introducing Eqs. (6) and (8) into the second equation in (7) yields

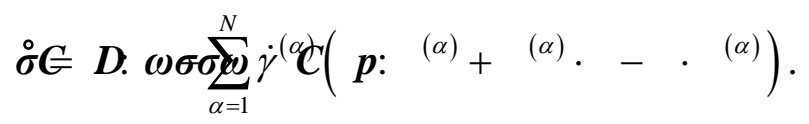

The crystalline slip is assumed to follow Schmid's law, and the slip rate $\dot{\gamma}^{(\alpha)}$ is assumed to be given by a visco-plastic power law using Schmid's resolved shear stress $\tau^{(\alpha)}$ as

$$
\frac{\dot{\gamma}^{(\alpha)}}{\dot{\gamma}_{0}}=\left|\frac{\tau^{(\alpha)}-\tau_{\mathrm{kin}}^{(\alpha)}}{\tau_{\mathrm{Y}}^{(\alpha)}}\right|^{\frac{1}{m}} \operatorname{sign}\left(\tau^{(\alpha)}-\tau_{\mathrm{kin}}^{(\alpha)}\right), \quad \tau^{(\alpha)}=\mathbf{s}(\alpha) \boldsymbol{m} \quad . \quad{ }^{(a)}, \quad \dot{\tau}_{\mathrm{Y}}^{(a)}=\sum_{\beta} q_{\alpha \beta} h\left|\dot{\gamma}^{(\beta)}\right|,
$$

where $\tau_{\text {kin }}^{(\alpha)}$ is the resolved back stress of the $\alpha$ slip system, $\tau_{\mathrm{Y}}^{(\alpha)}$ is the current strength of the $\alpha$ slip system with $\tau_{\mathrm{Y}}^{(\alpha)}=\tau_{0}$ initially, $\dot{\gamma}_{0}$ is a reference strain rate, $m$ is the rate sensitivity exponent, and $q_{\alpha \beta}$ is the self ( $\alpha=\beta$ ) and latent $(\alpha \neq \beta$ ) hardening moduli. The rate of hardening is $h$, and the hardening laws will be explained in the next section.

The resolved back stress $\tau_{\text {kin }}^{(\alpha)}$ is given in the form (Harder, 1999; Xie et al., 2004; Nakamachi et al., 2007)

$$
\tau_{\text {kin }}^{(\alpha)}=\mathbf{s} \stackrel{(\alpha)}{\boldsymbol{m}} \boldsymbol{m} \boldsymbol{F}_{\text {kin }} \cdot \stackrel{(\mathrm{a})}{ }, \quad{ }_{\text {kin }}=\sum_{\alpha} \Omega^{(\alpha) \quad(\alpha)},
$$

where $\sigma_{\text {kin }}$ is the back stress tensor and $\Omega^{(\alpha)}$ is the scalar kinematic variable of the $\alpha$ slip system.

The evolution of $\Omega^{(\alpha)}$ due to the short-range dislocation interaction is assumed as

$$
\dot{\Omega}^{(\alpha)}=c \dot{\gamma}^{(\alpha)}-d \Omega^{(\alpha)}\left|\dot{\gamma}^{(\alpha)}\right|,
$$

where $c$ and $d$ are the direct hardening and dynamic recovery coefficients, respectively. Because a hardening behavior of slip system is different depending on the family of slip systems in hcp 
metals, the coefficients $c$ and $d$ may also be different depending on the family of slip systems. However their identifications for each family are presently very difficult owing to the lack of knowledge on the cyclic loading behavior. Therefore for the sake of simplicity the coefficients $c$ and $d$ are assumed to be the same regardless of the family of slip systems.

\subsection{Treatment of Slip and Twinning Systems}

It is of great importance, in particular for hcp metals, to select appropriate families of slip and twinning systems to carry out a realistic crystal-plasticity analysis. Many studies have investigated the deformation mechanisms in Mg and its alloys experimentally (e.g., Reed-Hill and Robertson, 1958; Yoshinaga and Horiuchi, 1964) and numerically (e.g., Styczynski et al., 2004; Agnew et al., 2003). Surveys of the literature can be found in references Lou et al. (2007) and Graff et al. (2007). Graff et al. (2007) concluded based on their literature survey that the use of families of basal slip, any $<\mathrm{a}>$ slip and one $<\mathrm{a}+\mathrm{c}>$ slip, and tensile twinning systems seems to be necessary although the deformation mechanisms in $\mathrm{Mg}$ and its alloys are still open to discussion. In this study, three families of slip systems — basal $<\mathrm{a}>$ slip, prismatic $<\mathrm{a}>$ slip, and pyramidal-2 $<\mathrm{a}+\mathrm{c}>$ slip — and one family of $\left\{\begin{array}{llll}1 & 0 & \overline{1} & 2\end{array}\right\}$ tensile twinning systems are used to model the mechanical behavior of a Mg alloy sheet. There are 3 basal, 3 prismatic, 6 pyramidal-2, and 6 twinning systems. Table 1 shows the slip/twinning plane normal and slip direction vectors for these systems.

Depending on the family of slip systems, two evolution laws are used for the rate of hardening $h$ in the forms (Graff et al., 2007) 
Table 1. Plane normal and slip direction vectors of slip and twinning systems used in the present study.

\begin{tabular}{|c|c|c|c|}
\hline & Slip/twinnin & plane & $\begin{array}{l}\text { Slip direction/shear direction } \\
\text { due to twinning }\end{array}$ \\
\hline Basal & $\left(\begin{array}{lll}0 & 0 & 0\end{array}\right.$ & 1) & $\left\langle\begin{array}{llll}1 & 1 & \overline{2} & 0\end{array}\right\rangle$ \\
\hline Prismatic & $\left\{\begin{array}{lll}1 & 0 & \overline{1}\end{array}\right.$ & $0\}$ & $\left\langle\begin{array}{llll}1 & 1 & \overline{2} & 0\end{array}\right\rangle$ \\
\hline Pyramidal-2 & $\left\{\begin{array}{lll}1 & 1 & \overline{2}\end{array}\right.$ & $2\}$ & $\left\langle\begin{array}{llll}1 & 1 & \overline{2} & 3\end{array}\right\rangle$ \\
\hline Twinning & $\left\{\begin{array}{lll}1 & 0 & \overline{1}\end{array}\right.$ & $2\}$ & $\left\langle\begin{array}{llll}1 & 0 & \overline{1} & 1\end{array}\right\rangle$ \\
\hline
\end{tabular}

$$
\begin{aligned}
& h=h_{0}, \\
& h=h_{0}\left(1-\frac{\tau_{0}}{\tau_{\infty}}\right) \exp \left(-\frac{h_{0} \bar{\gamma}}{\tau_{\infty}}\right),
\end{aligned}
$$

where $\bar{\gamma}$ is the cumulative shear strain on all the slip systems and is given by

$$
\bar{\gamma}=\sum_{\alpha} \int\left|\dot{\gamma}^{(\alpha)}\right| \mathrm{d} t
$$

Linear hardening (Eq. (13)) is assumed for the basal slip, and Voce hardening (Eq. (14)) for the prismatic and pyramidal-2 slip.

As for a tensile twinning model, we employ the method first proposed by Van Houtte (1978) and then employed for Mg alloys by researchers (e.g., Staroselsky, et al., 2003; Mayama, et al., 2009). Twinning is assumed to have a polar character, where each system can be activated only by tension of the $c$-axis, and is modeled as follows. When a grain is twined entirely, it undergoes the shear strain due to twinning $\gamma_{\text {ref }}$. On the other hand, when part of a grain is twined, the shear strain arises in the grain can be approximately given as a function of the volume fraction of the twins if we assume that the shear in the grain is uniform (Chin, et al., 1969; VanHoutte, 1978). Using this approximation, the shear strain that arises in a grain due to twinning is 
given by $\bar{\gamma}_{\text {twin }}^{(\alpha)}=f^{(\alpha)} \gamma_{\text {ref }}$, where $f^{(\alpha)}$ is the volume fraction of the $\alpha$ twinning system, and $\bar{\gamma}_{\text {twin }}^{(\alpha)}$ is the cumulative shear strain on the $\alpha$ twinning system. Because this shear is similar to the shear caused by an activated slip system, the shear strain rate induced by twinning can be calculated in the similar way as that for slip deformation using eq. (10). Equation (13) is used for the rate of hardening $h$.

The lattice rotation due to twinning is modeled as follows. A pseudo-random threshold value $f_{\text {th }}^{(\alpha)}$ is initially determined between 0.3 and 1.0 for each twinning system (Staroselsky, et al., 2003; Mayama, et al., 2009). During calculation, the volume fraction of the $\alpha$ twinning system $f^{(\alpha)}$ is compared with $f_{\text {th }}^{(\alpha)}$ at each time increment, and if $f^{(\alpha)} \geq f_{\text {th }}^{(\alpha)}$ is satisfied, the entire grain is rotated to a specific twinning orientation. The lattice rotation tensor due to twinning $\boldsymbol{R}^{\mathrm{tw}}$ is given in the form

$$
\boldsymbol{R}^{t w}=2 \boldsymbol{m}^{(\alpha)} \otimes \boldsymbol{m}^{(\alpha)}-\boldsymbol{I},
$$

where $\boldsymbol{I}$ is the unit tensor, and $\boldsymbol{m}^{(\alpha)}$ is the plane normal vector for the $\alpha$ twinning system.

In this study, we assume that untwinning can be ignored, although this assumption may lead to a poor prediction accuracy of the inelastic behavior. This assumption is mainly because there is no well-established numerical model that can be used to predict the strain-path change behavior including the untwinning of Mg alloys (Proust et al., 2009). On the other hand, because the uniaxial tension of a rolled $\mathrm{Mg}$ alloy sheet is to be calculated in this study as will be described below, the activation of twinning may be small. Furthermore, we will focus on the effects of basal and nonbasal slip on the inelastic behavior, and the untwinning assumption will lead to a clearer understanding of these effects. 
The above rate-dependent crystal-plasticity model is incorporated into each Gauss point in the static finite-element method. The rate tangent modulus method (Pierce et al., 1983) is used for the explicit time integration of the constitutive model. The material parameters used in Eqs. (10), (13) and (14) will be determined in the following sections. Using the twinning model described above, $\gamma_{\text {ref }}$ can also be viewed as a material parameter that should be determined, and will also be determined in the following sections.

\section{Experimental Procedure}

To determine the material parameters described above and to verify the simulation results, monotonic uniaxial tensile and compressive tests and a loading-unloading test under uniaxial tension were carried out using a commercial rolled Mg alloy sheet of AZ31B (Mg-3\%Al-1\%Zn) with a thickness of $0.8 \mathrm{~mm}$ produced by the Osaka Fuji Corporation. The material was annealed at $350{ }^{\circ} \mathrm{C}$ for $1.5 \mathrm{~h}$ to obtain an $\mathrm{O}$ temper before the experiment. Tensile specimens were machined parallel to the rolling direction. The experiment was carried out at room temperature. A strain gauge (Kyowa Electronic Instruments Co., KFEM) was used to measure the strains during loading and unloading. The tests were carried out at an initial strain rate of $0.0007 \mathrm{~s}^{-1}$. The experimental data were recorded by a data logger approximately every $10 \mathrm{~ms}$. It should be noted that the in-plane compressive test was carried out using comb-shaped tools to give compressive forces in the thickness direction of the specimen (Kuwabara et al., 2009).

\section{Material Modelling}

For a crystal-plasticity finite-element analysis, it is important to choose an appropriate finiteelement model in terms of realistic computational time and reasonable prediction of mechanical 
behavior. Diard et al. (2005) reported that a polycrystalline model with a simplified 138 orientations may be sufficient to obtain reasonable stress-strain curves for a given texture. Graff et al. (2007) showed that a finite-element model with 8 × 8 × $8=512$ eight-node brick elements, each having an individual crystallographic orientation, is sufficient to predict stress-strain curves for a textured $\mathrm{Mg}$ rolled sheet in a channel-die compression. The finite-element model used in the present study was a cube that had ten uniform eight-node isoparametric brick elements using selective reduced integration in each direction, as shown in Fig. 2. Thus, the total number of elements was $10 \times 10 \times 10=1000$. The same initial crystallographic orientation was assigned to

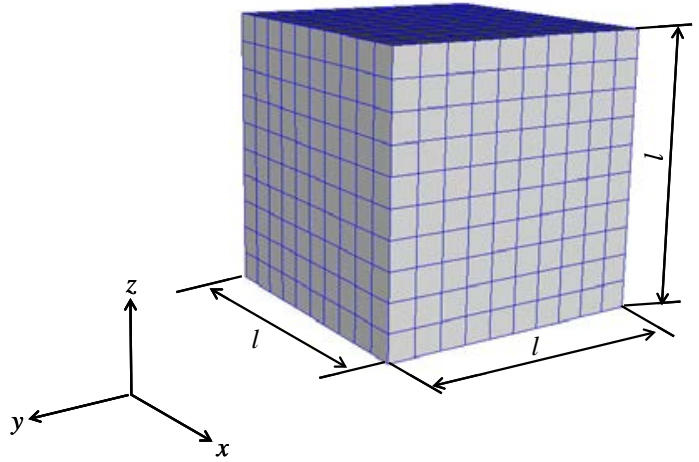

Fig. 2 Finite element model used in the simulation.

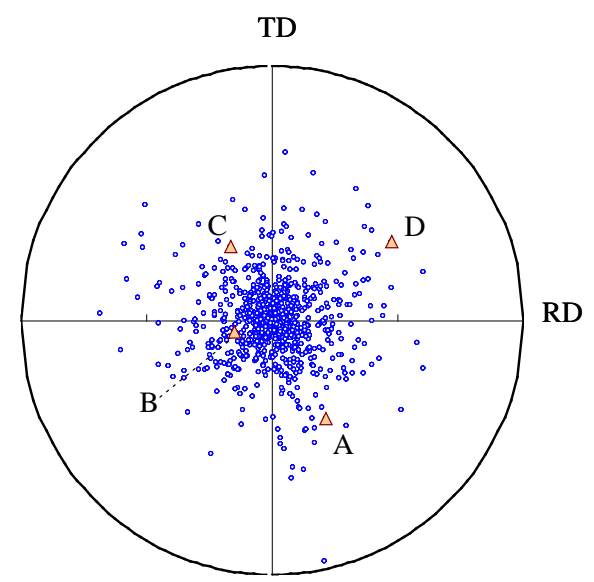

Fig. 3 (0001) pole figure used in the simulation model with a rolling texture.

all eight Gauss integration points in an element, thus the model had 1000 initial crystallographic orientations. Figure 3 shows the initial (0001) pole figure used in the simulation model. The initial crystallographic orientations were artificially created using the procedure proposed by Mayama et al. (2009) to simulate the rolling texture of Mg alloy sheets (Chino et al., 2008a; Graff et al., 2007; Agnew and Duygulu, 2005). 
A loading-unloading simulation under uniaxial tension along RD was carried out. The $x$-, $y$-, and z-axes in Fig. 2 were defined to be the rolling direction (RD), transverse direction (TD), and normal direction (ND), respectively, thus the majority of $c$-axes tended to align in the $z$ direction (ND). The following boundary conditions were used: the planes $x=0, y=0$, and $z=0$ were fixed in the $x, y$, and $z$ directions, respectively, and the displacement rate boundary conditions were given in RD, i.e., the plane $x=l$ was extended.

We assumed isotropic elasticity with Young's modulus $E=42 \mathrm{GPa}$ and Poisson ratio $v=0.3$ obtained by a uniaxial tensile test (Hama et al., 2010a) of the AZ31 Mg alloy sheet. The rate

Table 2. Latent-hardening parameters $q_{\alpha \beta}$ used in the present study (Graff et al., 2007) .

\begin{tabular}{ccccc}
\hline & Basal & Prismatic & Pyramidal-2 & Twinning \\
Basal & 0.2 & 0.5 & 0.5 & 0.5 \\
Prismatic & 0.2 & 0.2 & 0.2 & 0.5 \\
Pyramidal-2 & 1.0 & 1.0 & 0.2 & 0.25 \\
Twinning & 1.0 & 1.0 & 0.2 & 0.25 \\
\hline
\end{tabular}

sensitivity exponent was set to $m=0.02$ and the reference strain rate to $\dot{\gamma}_{0}=0.001 \mathrm{~s}^{-1}$. The choice of $m$ will be discussed in Section 5.1. The self-hardening parameters were set to 1, while the latent-hardening parameters $q_{\alpha \beta}$ shown in Table 2 were adopted; these were taken from the literature (Graff et al., 2007).

The other hardening parameters used in Eqs. (13), and (14) were identified as follows. We used the hardening parameters indicated in the literature (Graff et al., 2007) as initial values. In the present model, the initial yield stress in the stress-strain curve under tension was determined primarily by $\tau_{0}$ of the basal and prismatic slip systems, and the hardening curve after yielding was determined primarily by $\tau_{\infty}$ and $h_{0}$ of the prismatic slip systems. The parameters of the 
pyramidal-2 slip systems affected in particular the hardening curve at high strains. The parameters of the tensile twinning systems affected the stress-strain curve under compression. Based on the above results, we first calibrated the parameters of the basal, prismatic, and pyramidal-2 slip systems by trial and error to achieve a reasonable fit with experimental stressstrain curve under monotonic uniaxial tension. Then the parameters of the tensile twinning systems including $\gamma_{\text {ref }}$ were calibrated to achieve a fit with the stress-strain curve under monotonic uniaxial compression. During this process, minor adjustments of other parameters were also carried out. The above processes were repeated several times and the parameters were finally determined as shown in Table 3. $\gamma_{\text {ref }}$ was 0.1 . The coefficients of kinematic hardening used in Eq. (12) were set to zero when determining the parameters shown in Table 3. The effect of kinematic hardening on the unloading behavior will be discussed in section 5.1. The simulated and experimental stress-strain curves are shown in Fig. 4. They are consistent with the literature (Graff et al., 2007; Proust, et al., 2009; Clausen et al., 2008; Lévesque et al., 2010) with regard to the rank order of the initial CRSS values and the fact that the easiest and hardest slip modes are

(b) Logarithmic strain
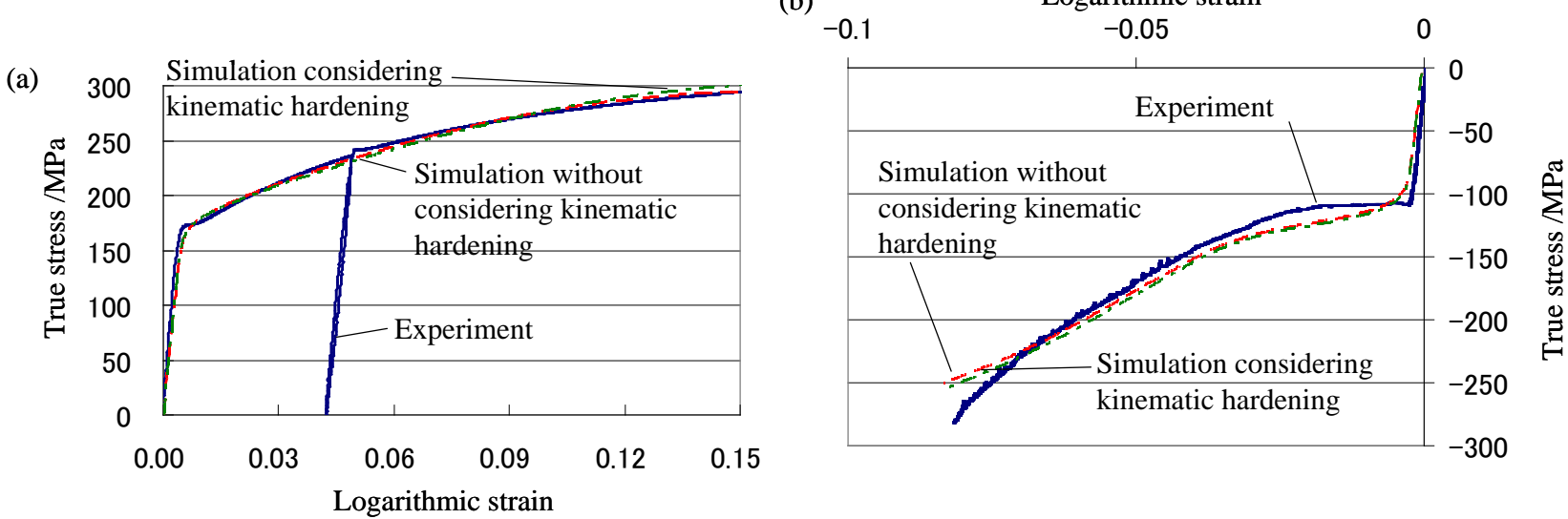

Fig. 4 Stress-strain curves obtained by the simulation: (a) uniaxial tension and (b) uniaxial compression. 
Table 3. Calibrated material parameters in Eqs. (13) and (14).

\begin{tabular}{ccccc}
\hline & Basal & Prismatic & Pyramidal-2 & Twinning \\
$\tau_{0}$ & 10 & 85 & 160 & 48 \\
$\tau_{\infty}$ & - & 260 & 360 & - \\
$h_{0}$ & 10 & 600 & 750 & 150 \\
\hline
\end{tabular}

basal $<\mathrm{a}>$ and pyramidal- $2<\mathrm{a}+\mathrm{c}>$, respectively. The tendency in the simulated stress-strain curve under monotonic compression is different especially at strains higher than -0.07 . This difference may be owing to the fact that some mechanisms that may influence the stress-strain curve at large strain are not taken into account in the model. One of them may be compressive twinning systems that are activated at high strains (Chino et al., 2008b). On the other hand, because the loading-unloading behavior under uniaxial tension up to a strain of about $3 \%$ is analyzed in detail in this study as will be described below, the effect of this difference on our results may be negligible.

To see the sensitivity of mesh discretization and initial crystallographic orientations to the stress-strain curve, Fig. 5 shows the stress-strain curves obtained using five sets of initial rolling textures for various numbers of elements. Five sets of initial rolling textures used here were

(a)

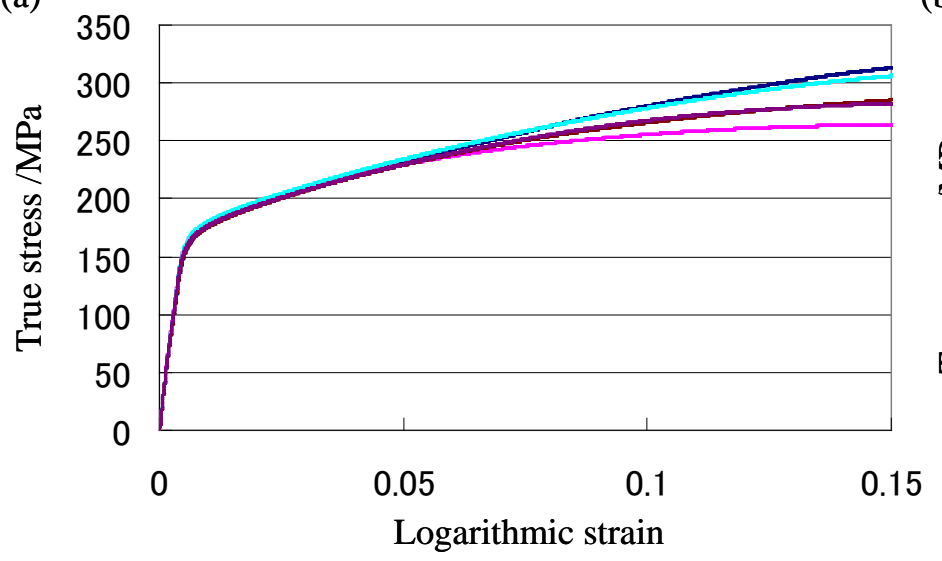

(b)

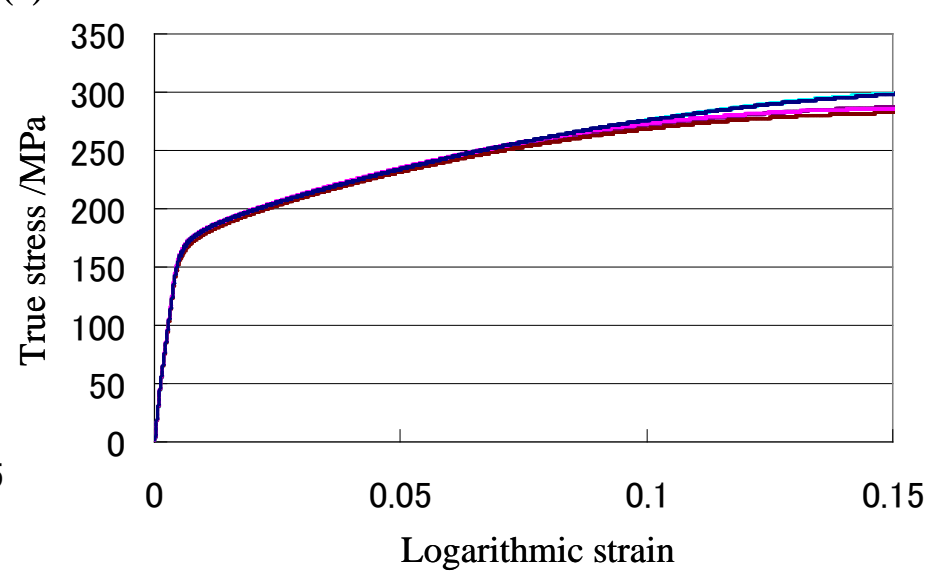


(c)

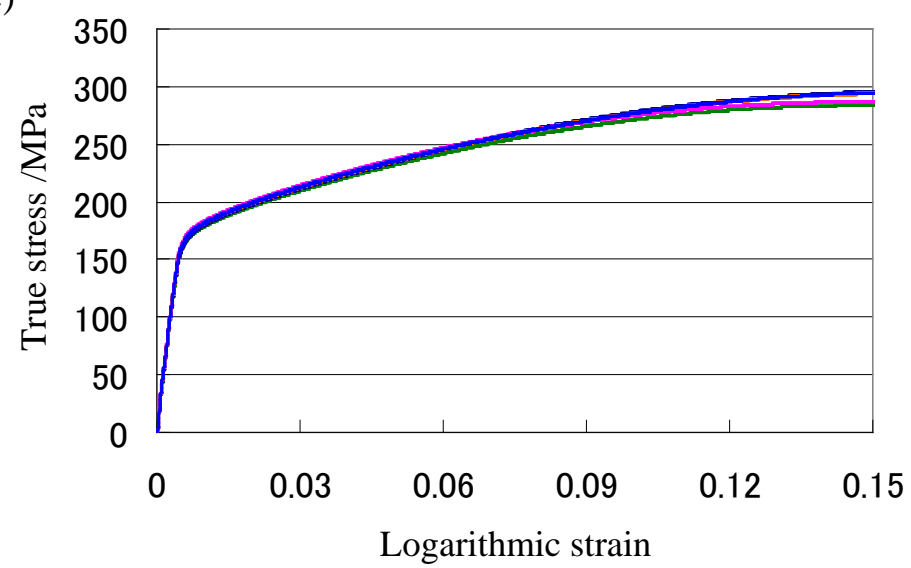

Fig. 5 Stress-strain curves obtained using five sets of initial orientations with various numbers of elements. (a) 125 (=5x5x5) elements, (b) 343 (=7x7x7) elements, and (c) 1000 (=10x10x10) elements.

(a)

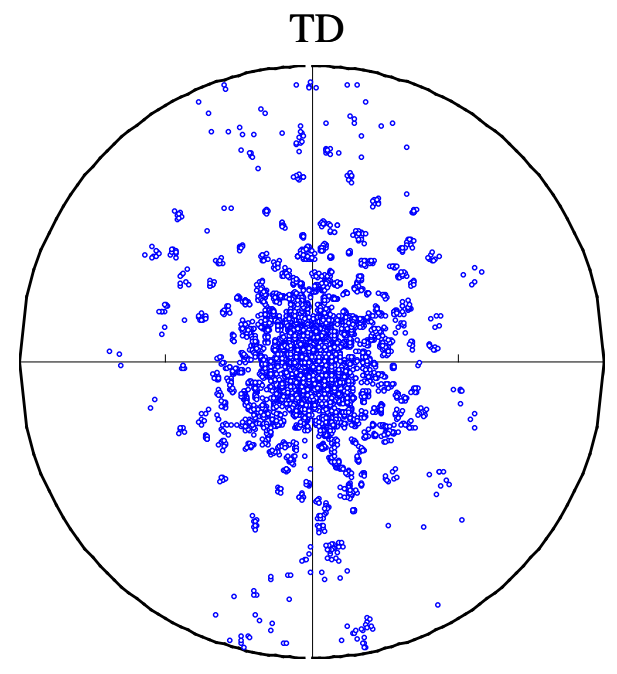

(b)

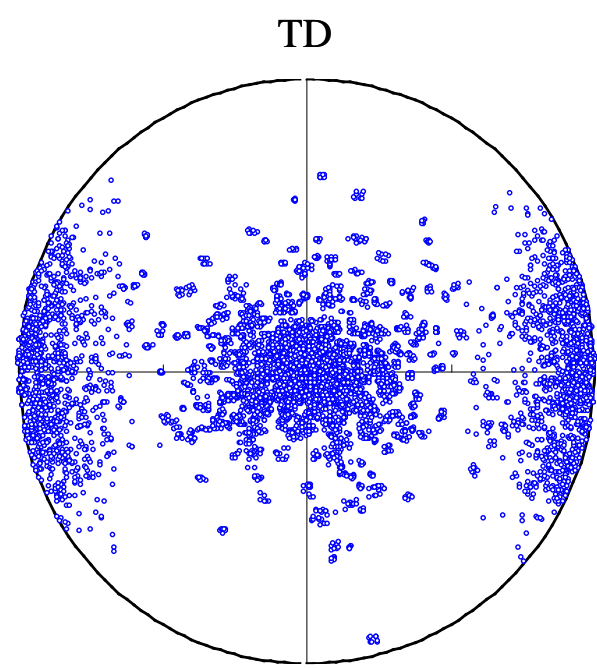

$\mathrm{RD}$

Fig. 6 (0001) pole figures in various deformation stages: (a) Texture after tension along RD (0.1 tensile strain), (b) Texture after compression along RD (0.07 compressive strain). 
created by changing pseudo-random numbers (Mayama et al., 2009). The stress-strain curve tends to saturate as the number of elements increases, and the curves obtained with 1000 elements are almost the same up to a strain of about $9 \%$. This result is consistent with the literature (Tadano, 2010). From the above results, we can reasonably say that our present model is acceptable to investigate the deformation up to a strain of about $3 \%$.

Figure 6 shows the (0001) pole figures in various deformation stages obtained by the simulation. Both results are qualitatively in good agreement with the literature (Lou, et al., 2007).

Although the above results show the validity of the calibrated hardening parameters shown in Table 3, it should be noted that we cannot guarantee that these parameters give the best fit and are unique for the material used in this study. For instance, a very similar stress-strain curve shown in Fig. 4 (a) can be obtained using another set of hardening parameters (Hama et al., 2010b), in which the hardening parameters for the pyramidal-2 slip systems are notably different. Nonetheless, activities of the slip and twinning systems obtained by this set of hardening parameters were qualitatively in good agreement with the present results that are shown below.

\section{Results and Discussion}

\subsection{Macroscopic Stress-Strain Curve}

The stress-strain curve obtained by the loading-unloading simulation under uniaxial tension is shown in Fig. 7. The simulation result shows clear inelastic behavior during unloading, although the magnitude of the hysteresis is smaller than in the experiment. Cyclic loading-unloading tests were carried out for various strains. The variations with the stress of the instantaneous gradient $\mathrm{d} \sigma / \mathrm{d} \varepsilon$ during unloading obtained by the experiment and the simulation are shown in Figs. 8 and 9 , respectively. The strain at the beginning of unloading is hereafter termed the unloaded strain. The 
gradient was calculated using two data points for every 100 successive data points in the experiment and for every data point in the simulation. The horizontal axes of Figs. 8 and 9 are the stress nondimensionalized by the stress at the beginning of unloading $\sigma_{0}$, and the vertical axes are the instantaneous gradient nondimensionalized by Young's modulus E.

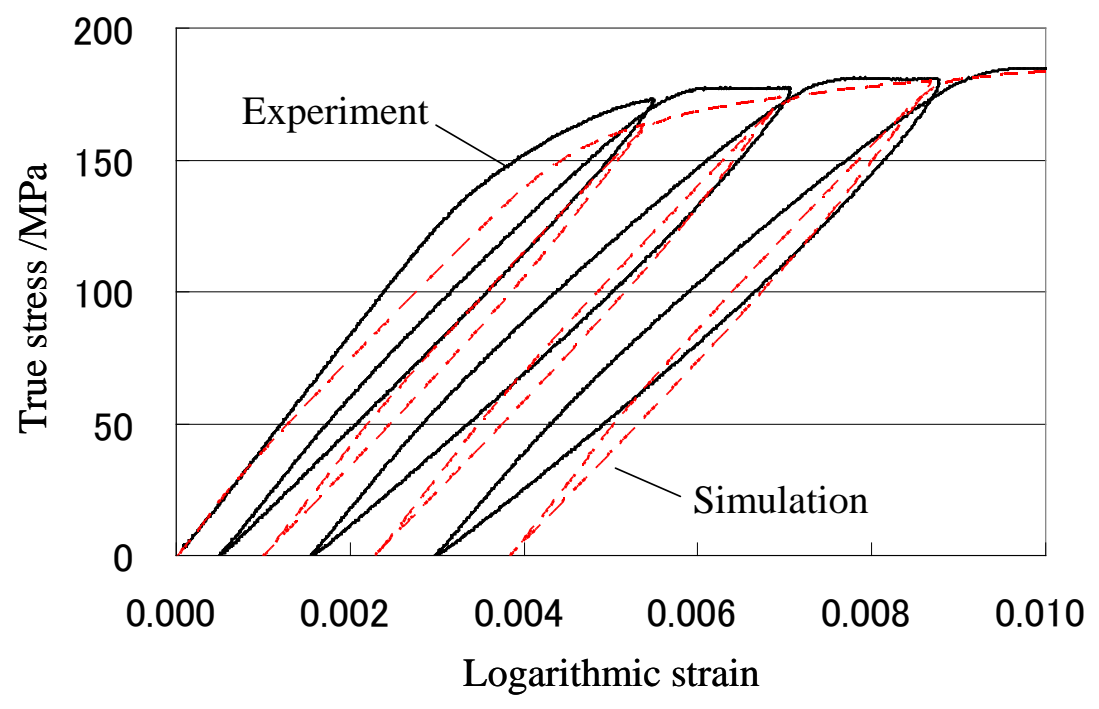

Fig. 7 Comparison of simulated and experimental inelastic behavior during unloading.

(a)

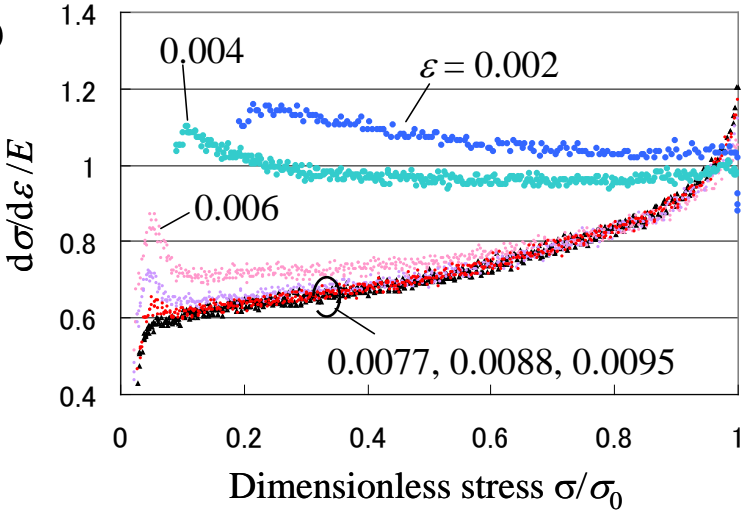

(b)

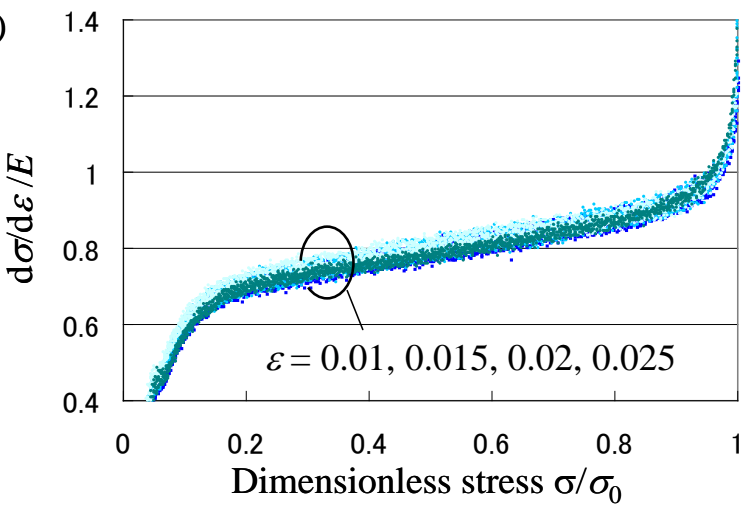

Fig. 8 Variation in instantaneous gradient $\mathrm{d} \sigma / \mathrm{d} \varepsilon$ during unloading obtained by the experiment in the unloaded strain range: (a) from 0.002 to 0.0095 and (b) from 0.01 to 0.025 . 
(a)

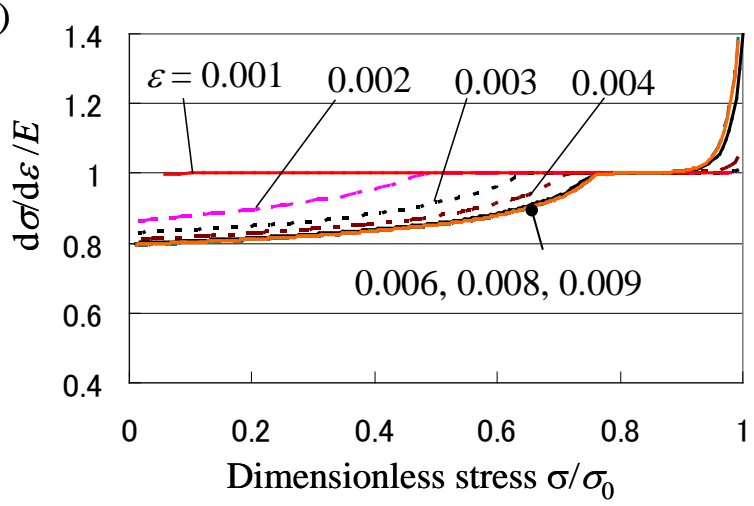

(b)

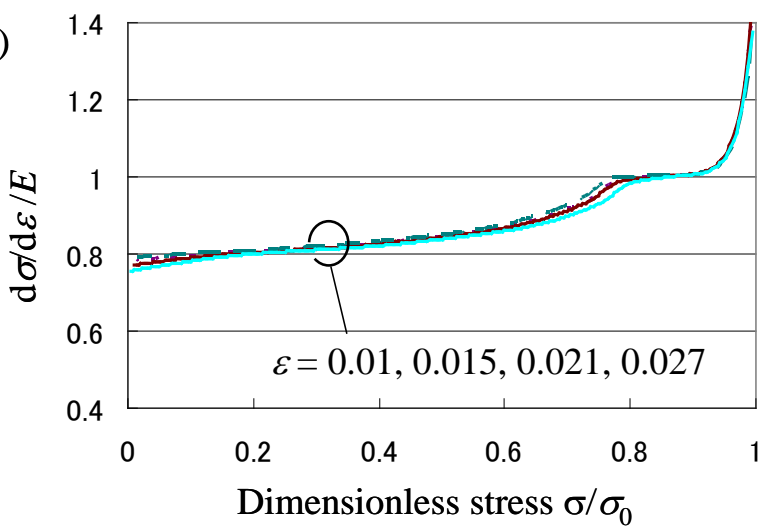

Fig. 9 Variation in instantaneous gradient during unloading obtained by the simulation for $m=0.02$ in the unloaded strain range: (a) from 0.001 to 0.009 and (b) from 0.01 to 0.027 .

The overall trend of the variation in the experiment (Fig. 8) is as follows. At unloaded strains of 0.002 and 0.004 , the instantaneous gradient is nearly constant during unloading because the sheet is still almost elastic (Fig. 8 (a)). As the unloaded strain increases above 0.004, the variation changes as follows: the instantaneous gradient decreases rapidly at the beginning of unloading, then decreases only slightly at the middle stage, and finally decreases rapidly again at the end of unloading. A similar trend is observed in other materials (Cleveland and Ghosh, 2002). The amount of decrease in the instantaneous gradient at the middle stage becomes large as the unloaded strain increases, but this saturates at an unloaded strain of about 0.01 and the variation curve does not change thereafter (Fig. 8 (b)). A similar saturation trend has been reported in the literature (Cáceres et al., 2003; Mann et al., 2007).

In the simulation result (Fig. 9), the variation curve is not in good agreement with the experiment except at the very beginning where the instantaneous gradient decreases rapidly, and the magnitude of inelastic behavior is smaller than in the experiment. On the other hand, the observations that the decrease in the instantaneous gradient becomes large as the unloaded strain 
increases (Fig. 9 (a)) and the variation curve saturates at an unloaded strain of about 0.01 (Fig. 9 (b)) are in good agreement with the experiment. This shows that the simulation results are qualitatively acceptable.

There may be several reasons for these inconsistencies between the experiment and simulation results, including untwinning, kinematic hardening of the slip systems and the simple boundary conditions given to the sheet model. To investigate the effect of kinematic hardening on the variation curve numerically, the coefficients for kinematic hardening used in Eq. (12) were set to $c=200$ and $d=30$ and the hardening parameters were re-characterized accordingly as shown in Table 4. The stress-strain curves obtained considering kinematic hardening are shown in Fig. 4. Cyclic loading-unloading tests were carried out considering kinematic hardening and the variations with the stress of the instantaneous gradient $\mathrm{d} \sigma / \mathrm{d} \varepsilon$ during unloading are shown in Fig. 10. The result obtained considering kinematic hardening gives close agreement with that without considering kinematic hardening. The simulations were carried out using several sets of

(a)

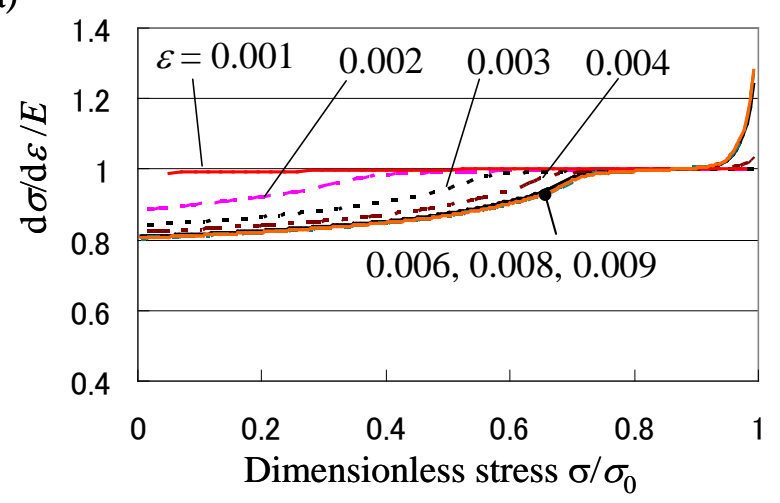

(b)

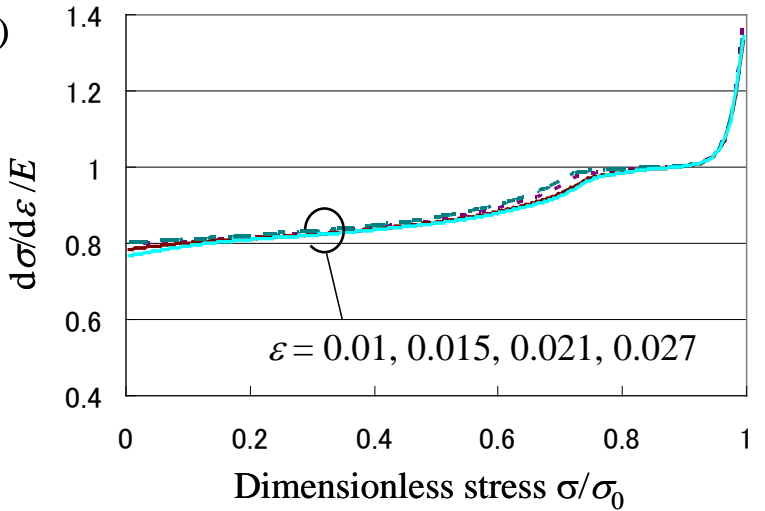

Fig. 10 Variation in instantaneous gradient during unloading obtained by the simulation considering kinematic hardening for $m=0.02$ in the unloaded strain range: (a) from 0.001 to 0.009 and (b) from 0.01 to 0.027 . 
Table 4. Calibrated material parameters in Eqs. (13) and (14) with the coefficients of kinematic hardening $c=200$ and $d=30$.

\begin{tabular}{ccccc}
\hline & Basal & Prismatic & Pyramidal-2 & Twinning \\
$\tau_{0}$ & 5 & 78 & 160 & 48 \\
$\tau_{\infty}$ & - & 280 & 360 & - \\
$h_{0}$ & 10 & 500 & 750 & 150 \\
\hline
\end{tabular}

coefficients for kinematic hardening, in which $c$ was ranging from 0 to 200 and $d$ was characterized accordingly, but the result remained almost the same. However we cannot conclude that kinematic hardening does not play an important role in the unloading behavior in this simulation condition solely from the above results because the kinematic hardening model employed in this study was very simplified as explained in section 2.2 and the coefficients for kinematic hardening should be in fact determined from a cyclic stress-strain curve. Further investigation on the effect of kinematic hardening is necessary using a more sophisticated kinematic hardening model. This will be future work.

To examine the effect of the rate sensitivity exponent $m$, the simulations were also carried out for $m=0.1$ and 0.0005 . Figures 11 and 12 show the variations in the instantaneous gradient with the stress during unloading for $m=0.1$ and 0.0005 , respectively. The variation at the initial stage apparently depends on the value of $m$ : the strain range in which the instantaneous gradient rapidly decreases becomes small as $m$ decreases and becomes negligible at $m=0.0005$. The variation curves at the middle and end of unloading are almost independent of the value of $m$. We selected $m=0.02$ because, of the above three values, this value gives the most appropriate variation curve for the sheet material and the experimental conditions. 
(a)

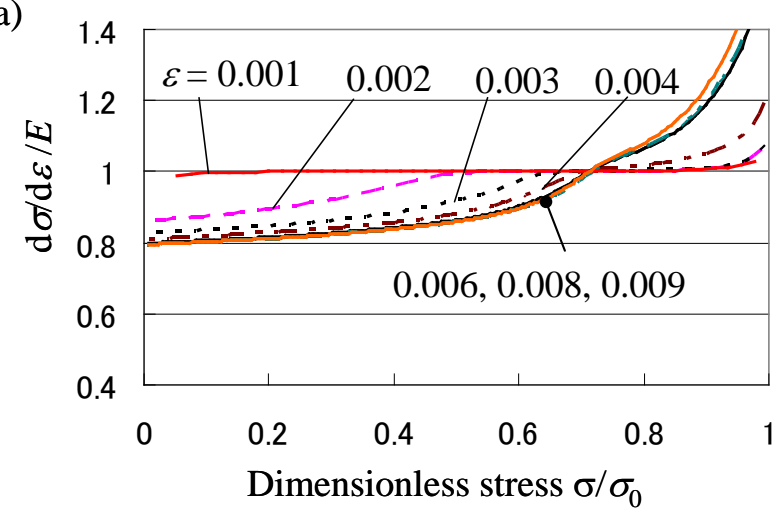

(b)

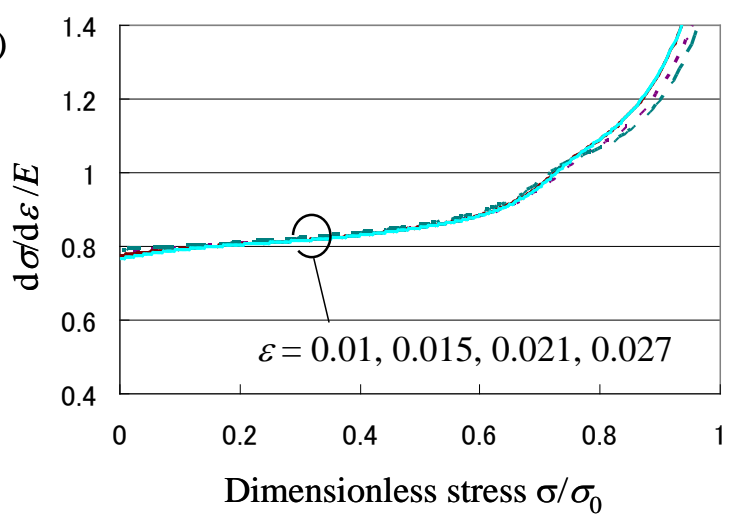

Fig. 11 Variation in instantaneous gradient during unloading obtained by the simulation for $m=0.1$ in the unloaded strain range: (a) from 0.001 to 0.009 and (b) from 0.01 to 0.027 .

(a)

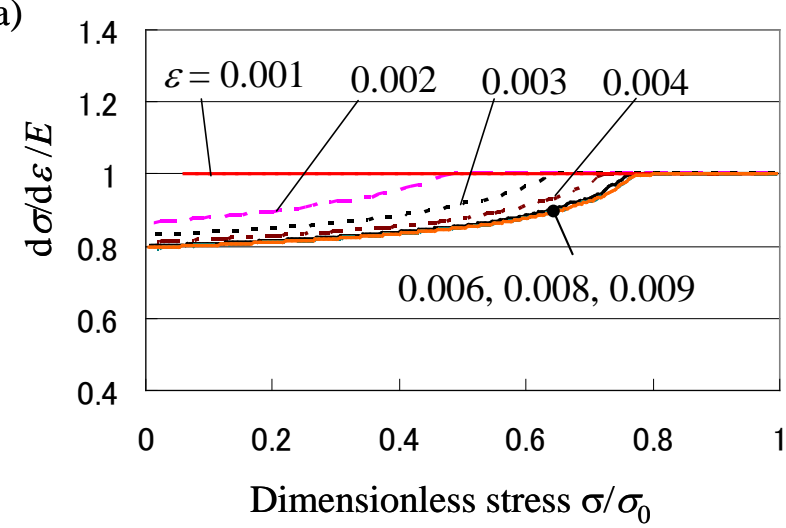

(b)

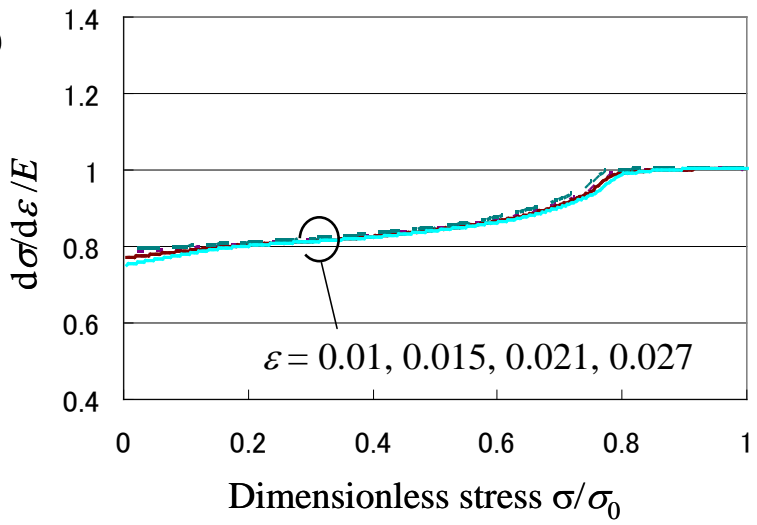

Fig. 12 Variation in instantaneous gradient during unloading obtained by the simulation for $m=$ 0.0005 in the unloaded strain range: (a) from 0.001 to 0.009 and (b) from 0.01 to 0.027 .

\subsection{Activity of Slip Systems}

In the following, the mechanism of the inelastic behavior during unloading is examined from a mesoscopic point of view using the simulation results. The evolution of the activities of each family of slip systems during loading and unloading is first investigated. The activity of each 
family of slip systems $i$ is evaluated by the plastic strain increment contributed by the family of slip systems i, summed over all grains in the form (Tomé et al., 1991):

$$
r_{\mathrm{i}}=\sum_{\mathrm{n}(\text { allgrains) }} \sum_{\substack{\text { (afamily of } \\ \text { slipsystems,i })}}\left|\Delta \gamma^{(\mathrm{n}, \mathrm{k})}\right|,
$$

where $\mathrm{n}$ is the number of grains; and $\mathrm{k}$ is the number of slip systems of the family $\mathrm{i}$. It should be noted that the so-called relative activity is not used in this study because the magnitude of plastic strain increment varies drastically during loading and unloading. The simulated evolution of the plastic strain increment of each family with logarithmic strain during monotonic uniaxial tension is shown in Fig. 13 for reference. The activation of the basal slip systems is dominant initially. The prismatic slip systems are then activated and the activations of these two families become dominant during the deformation. On the other hand, the activations of the tensile twinning systems and the pyramidal-2 slip systems are small throughout the deformation. Owing to twin reorientation, the plastic strain increments fluctuate instantaneously at some strains higher than about $6 \%$.

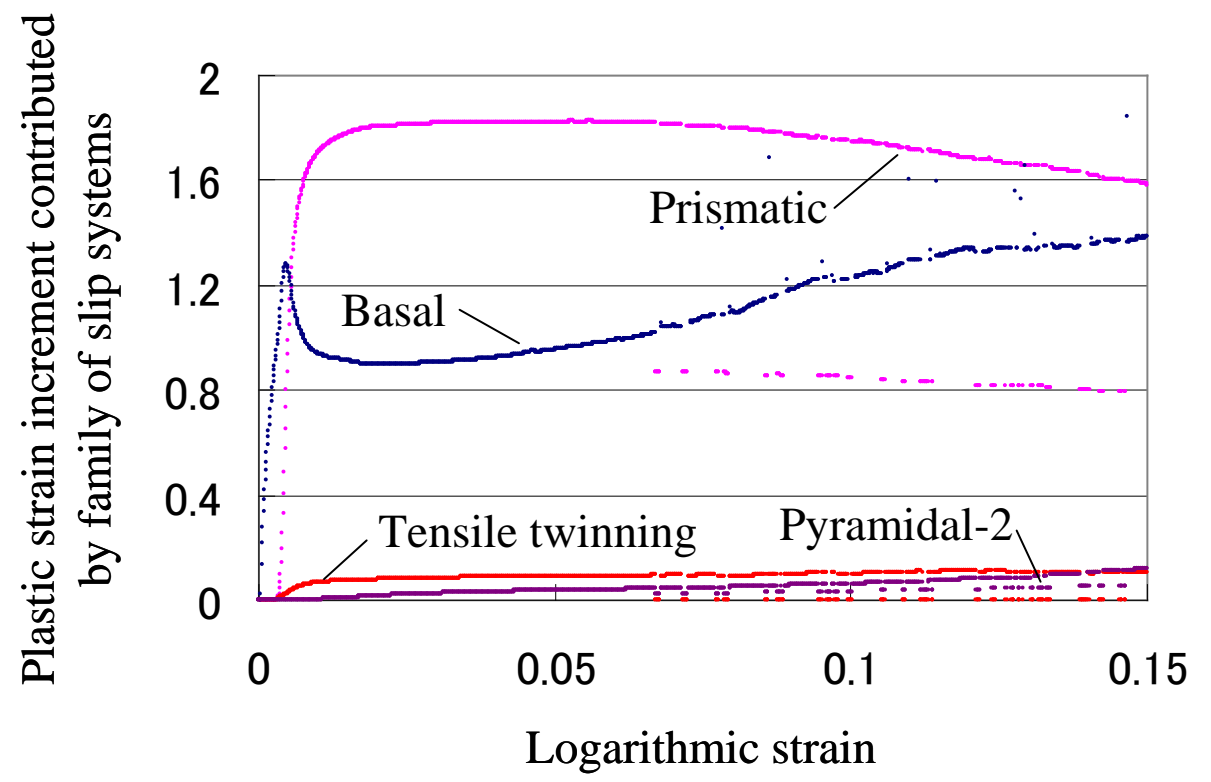

Fig. 13 Plastic strain increment contributed by family of slip systems during uniaxial tension. 


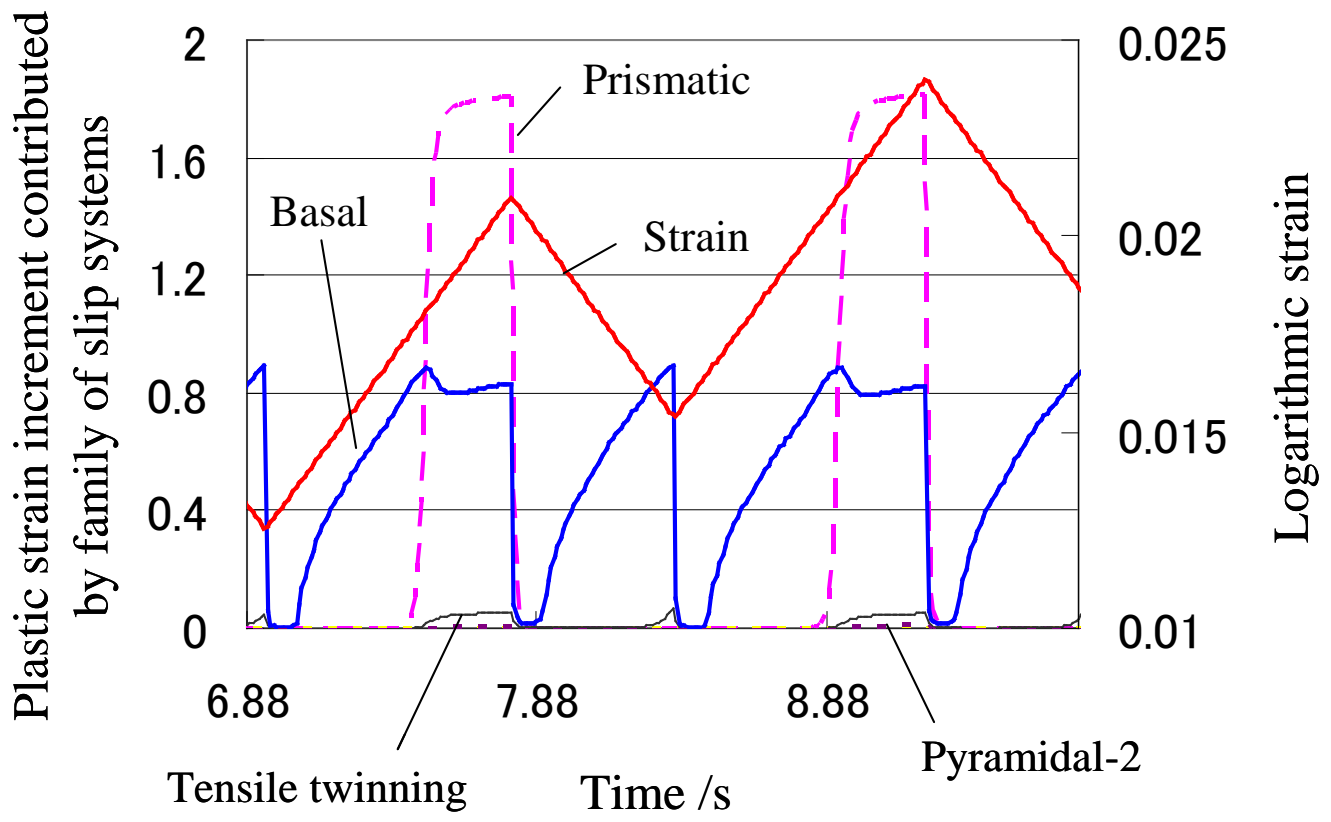

Fig. 14 Plastic strain increment contributed by family of slip systems during loading-unloading in the strain range from 0.012 to 0.024 .

Figure 14 shows the simulated evolution of the plastic strain increment during the loadingunloading as a function of time. In Fig. 14, the sheet is unloaded every strain of 0.003 and the figure shows the evolution in the strain range between 0.012 and 0.024 , during which two loading-unloading cycles occurred. The activation of the basal slip systems is dominant initially, and then the prismatic slip systems start to be activated. A similar tendency is also observed at the beginning of the monotonic uniaxial tension (Fig. 13). When the sheet starts to be unloaded, the activation of the prismatic slip systems suddenly vanishes and the activation of the basal slip systems again becomes dominant. The activation of the pyramidal-2 slip system is negligible during unloading. These trends remain unchanged during unloading, and are the same for the two cycles. The tensile twinning systems are slightly activated when the loading direction is inverted. It should be noted that the twinning systems activated during loading are different from those activated during unloading. The above result shows that the basal slip and tensile twinning 
systems are activated at the very beginning of unloading, and the basal slip systems is primarily activated thereafter. This indicates that the inelastic behavior during unloading observed in the simulation is primarily due to the activation of the basal slip systems; the contributions of the prismatic slip, and pyramidal-2 slip systems are negligible. This also exhibits that the clear inelastic behavior shown in Fig. 9 can arise without considering untwinning although the magnitude of the hysteresis is smaller than in the experiment (Figs. 8 and 9).

Although the activity of the basal slip systems is large during both loading and unloading, the deformation mode may be different because the direction of the macroscopic displacement rate boundary conditions is opposite. Therefore, the sliding direction of the slip systems during the loading-unloading cycle is examined. The average sliding direction of each family of slip systems over all the grains is evaluated by

$$
r_{\mathrm{i}}^{\mathrm{s}}=\frac{\sum_{\mathrm{n}(\text { all grains })} \sum_{\substack{\text { (amainilyof } \\ \text { slipsystems,i })}} \Delta \gamma^{(\mathrm{n}, \mathrm{k})}}{\sum_{\mathrm{n} \text { (allgrains) }} \sum_{\substack{\text { (allfamilies of } \\ \text { slip systems }}} \mid \Delta \gamma^{(\mathrm{n}, \mathrm{j}) \mid}},
$$

whre the denominator is the plastic strain increment contributed by all the families, summed over

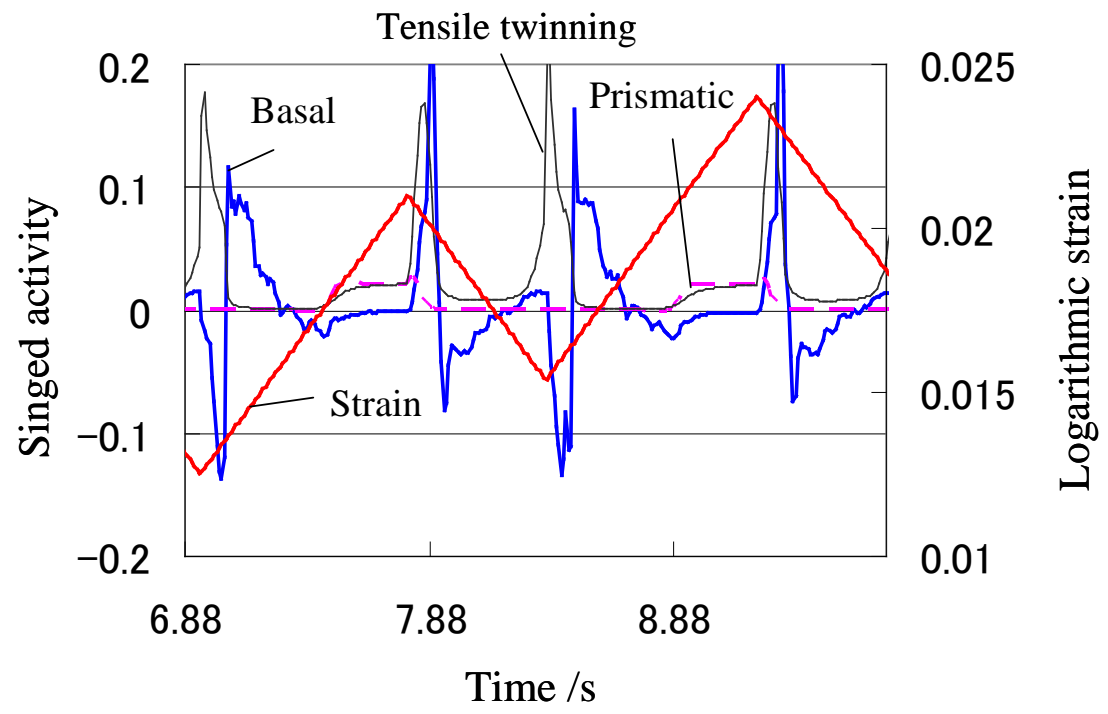

Fig. 15 Signed activities of families of slip systems during loading-unloading in the strain range from 0.012 to 0.024 . 
all the grains, and $\mathrm{j}$ is the total number of slip systems. Equation (18) is termed the signed activity. Figure 15 shows the evolution of the signed activities during the same period covered in Fig. 14. Note that the comparison of the magnitude of $r_{\mathrm{i}}^{\mathrm{s}}$ among the families given by Eq. (18) is meaningless because every slip increment $\Delta \gamma^{(n, k)}$ refers to a different local coordinate system. Therefore, we focus particularly on the signs of $r_{\mathrm{i}}^{\mathrm{s}}$.

During loading, the basal slip, prismatic slip, and tensile twinning systems are activated in the positive direction. When the loading direction is inverted, the activation of the prismatic slip systems suddenly vanishes, as also observed in Fig. 14. On the other hand, the basal slip systems are still activated during unloading, but the average sliding direction is negative. This shows that during unloading the basal slip systems are activated in a direction opposite to that during loading. The tensile twinning systems are also activated during unloading, but the direction is of course the same as during loading, because the untwinning is not taken into account in the present study.

\subsection{Evolution of Stress and Activity of Slip Systems in a Grain}

Figures 14 and 15 indicate that the partial reversal of the basal slip systems may be one of the causes of the inelastic behavior during unloading. To examine this hypothesis in more detail at the grain level, similar analyses were carried out for four representative integration points. Note that similar results were observed for many integration points, from which these points were arbitrarily chosen. The initial orientations of these points are indicated as A, B, C, and D in Fig. 3. The initial crystal local frame $\tilde{\boldsymbol{e}}_{i}$, which is schematically shown in Fig. 16, of the integration points is given in the global coordinate system shown in Fig. 2 as 


$$
\begin{aligned}
& \text { point A: } \tilde{\boldsymbol{e}}_{x}=\left\{\begin{array}{c}
-0.8836 \\
-0.0012 \\
0.4682
\end{array}\right\}, \quad \tilde{\boldsymbol{e}}_{y}=\left\{\begin{array}{c}
0.3011 \\
0.7643 \\
0.5703
\end{array}\right\}, \quad \tilde{\boldsymbol{e}}_{z}=\left\{\begin{array}{c}
0.3586 \\
-0.6449 \\
0.6750
\end{array}\right\}, \\
& \text { point B: } \tilde{\boldsymbol{e}}_{x}=\left\{\begin{array}{l}
-0.3062 \\
-0.9344 \\
-0.1819
\end{array}\right\}, \quad \tilde{\boldsymbol{e}}_{y}=\left\{\begin{array}{c}
-0.9064 \\
0.3446 \\
-0.2442
\end{array}\right\}, \quad \tilde{\boldsymbol{e}}_{z}=\left\{\begin{array}{c}
-0.2908 \\
-0.0901 \\
0.9525
\end{array}\right\}, \\
& \text { point C: } \tilde{\boldsymbol{e}}_{x}=\left\{\begin{array}{c}
-0.9527 \\
-0.2148 \\
-0.2149
\end{array}\right\}, \quad \tilde{\boldsymbol{e}}_{y}=\left\{\begin{array}{c}
-0.0595 \\
0.8255 \\
-0.5613
\end{array}\right\}, \quad \tilde{\boldsymbol{e}}_{z}=\left\{\begin{array}{c}
-0.2979 \\
0.5220 \\
0.7993
\end{array}\right\}, \\
& \text { point D: } \tilde{\boldsymbol{e}}_{x}=\left\{\begin{array}{c}
-0.1962 \\
0.8484 \\
-0.4917
\end{array}\right\}, \quad \tilde{\boldsymbol{e}}_{y}=\left\{\begin{array}{c}
0.6669 \\
0.2521 \\
-0.7012
\end{array}\right\}, \quad \tilde{\boldsymbol{e}}_{z}=\left\{\begin{array}{c}
0.7188 \\
-0.4655 \\
-0.5163
\end{array}\right\},
\end{aligned}
$$

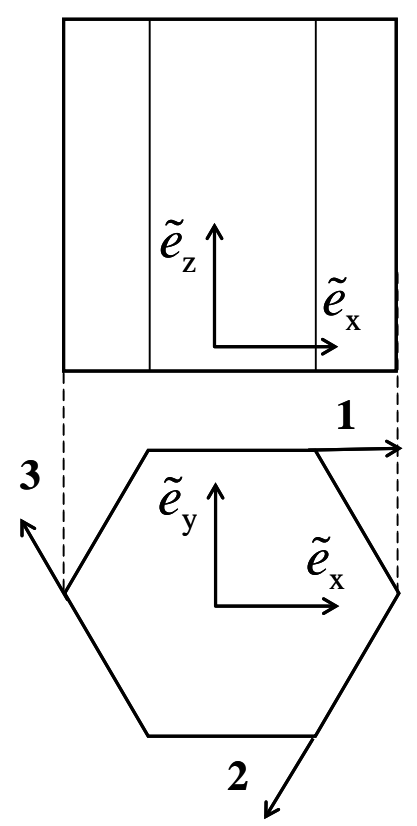

Fig. 16 Crystal local coordinate system $\tilde{\boldsymbol{e}}_{i}$. 

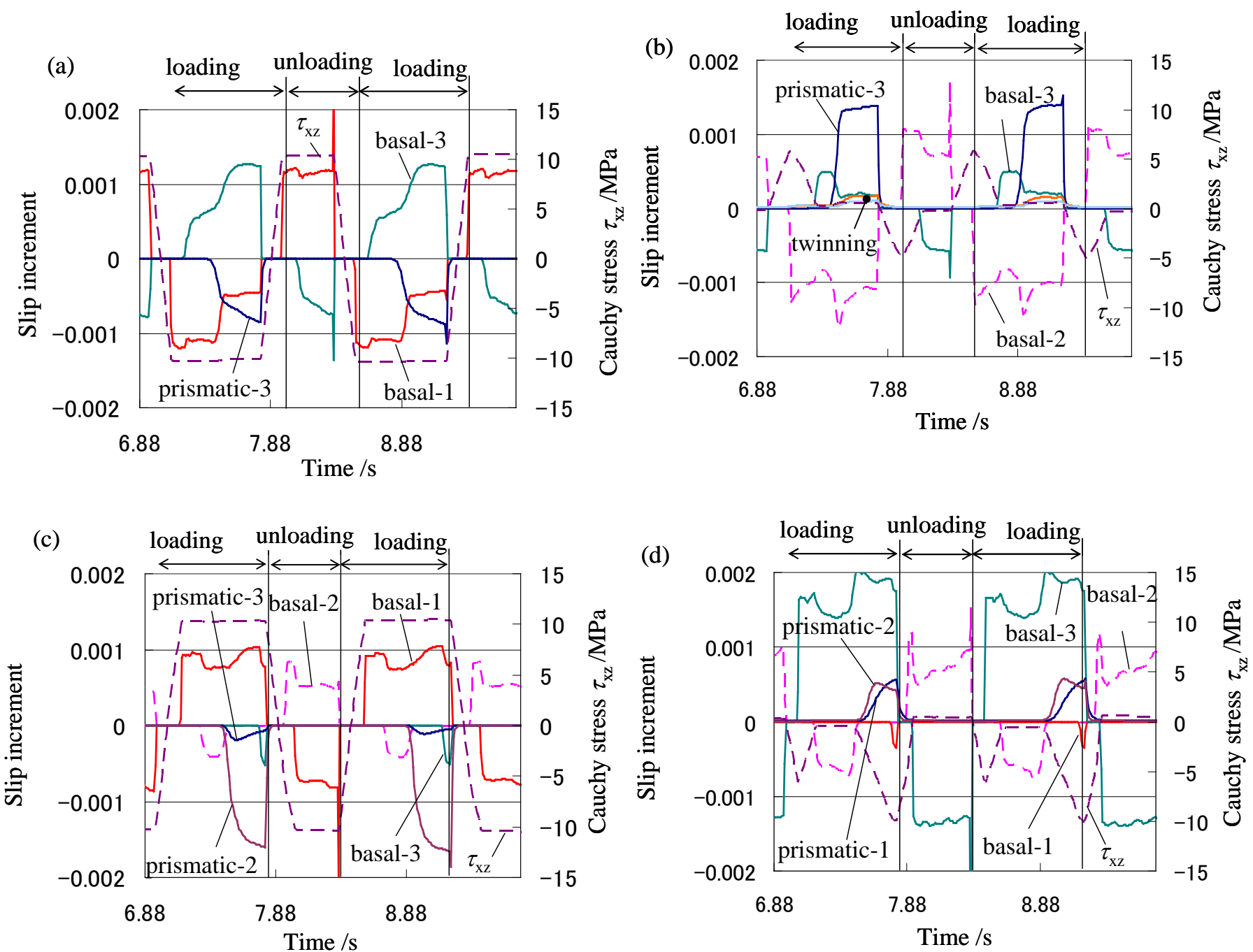

Fig. 17 Variations in slip increment in each slip system and Cauchy stress component $\tau_{\mathrm{Xz}}$. (a) Integration point A, (b) B, (c) C, and (d) D. 
The arrows 1, 2, and 3 in Fig. 16 indicate the positive slip directions of the basal and prismatic slip systems. Figure 17 shows the variation in the slip increment in each basal slip system during the same period covered in Fig. 14. For the point A, the basal-1 slip system is activated during both loading and unloading, but the slip direction is inverted with the inversion of the loading direction. Similarly, the basal-3 slip system is activated in the positive direction during loading and in the negative direction during unloading. The prismatic-3 slip system is activated during only loading. A similar tendency arises in the points B, C, and D. The tensile twinning systems are activated a little during only loading in point $\mathrm{B}$. The activation of the pyramidal-2 slip systems is negligible in the all points. Although the initial orientations are notably different in these points, a tendency observed is clearly similar to that shown in Figs. 14 and 15.

To examine the inversion of the slip direction of the basal-1 slip system, Fig. 18 shows the evolution of the Cauchy stress components at these integration points. The stress components refer to the local coordinate system $\tilde{\boldsymbol{e}}_{i}$ shown in Fig. 16, thus $\tau_{\mathrm{xz}}$ corresponds to Schmid's resolved shear stress of the basal-1 slip system. Most of the components increase and decrease alternately with the loading-unloading cycle. Some, including Schmid's resolved shear stress of the basal-1 slip system, $\tau_{\mathrm{xz}}$, even change their signs during loading and unloading. This trend is clear in points A and C. Such stress evolution in the grains may arise from the inhomogeneity of the material. Because the activation of the prismatic slip systems is dominant during loading as shown in Fig. 14, the overall stress level during loading is determined primarily by the prismatic slip systems. However the prismatic slip systems are hardly activated during unloading because the stress level during unloading is of course lower than that during loading. On the other hand, because the strength of the basal slip systems is much lower than that of the prismatic slip systems as shown in Table 3, they can be activated under the stress level during unloading. More 

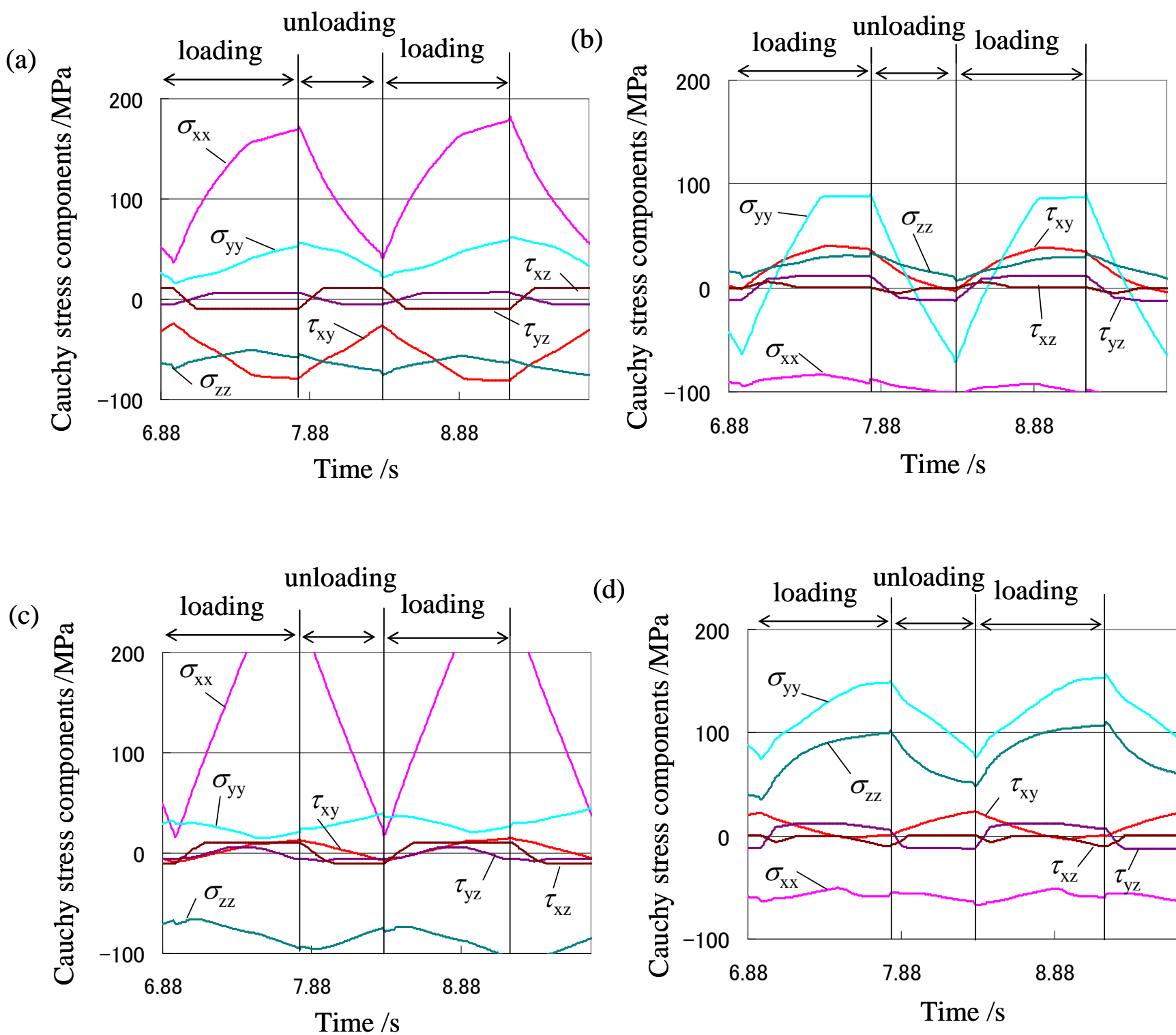

(d)

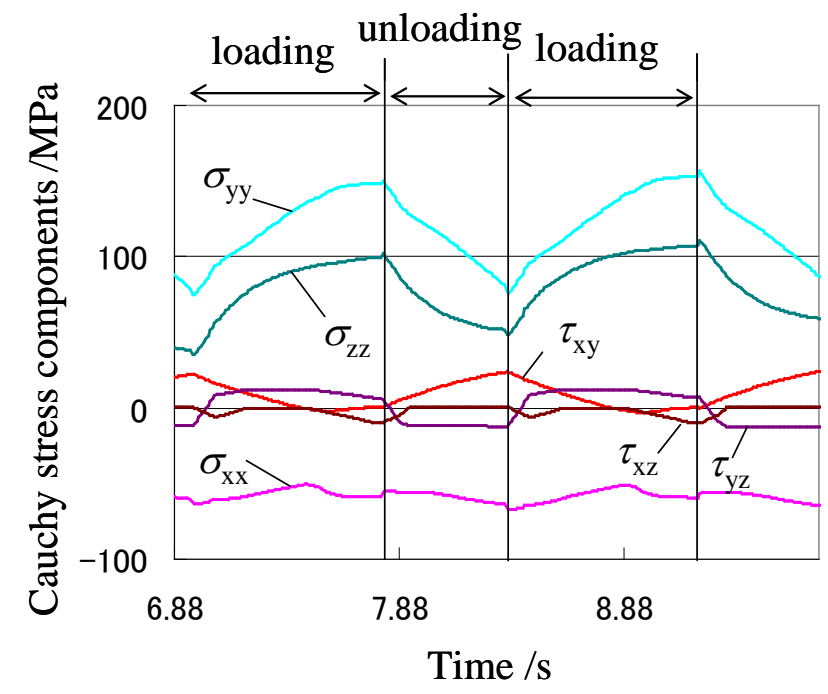

Fig. 18 Variations in Cauchy stress components in the local coordinate system $\tilde{\boldsymbol{e}}_{i}$. (a) Integration point A, (b) B, (c) C, and (d) D. 
precisely, the basal slip systems would be easily activated in the opposite direction when their Schmid's resolved shear stresses change sign during unloading. This is supported by the fact that the slip increment of the basal-1 system has a strong correlation with $\tau_{\mathrm{xz}}$, as shown in Fig. 17 (a) and (c). We conclude that one explanation for the inelastic behavior during unloading is that the basal slip systems are easily activated because their strength is much lower than that of the prismatic slip systems which are the dominant activated systems during loading. This also indicates that the effect of kinematic hardening of the slip systems on the unloading behavior should be examined in detail to further understand the mechanism as discussed in section 5.1.

\subsection{Effect of Initial Texture}

The above results indicate that the inelastic behavior during unloading is induced by the inhomogeneity of the material. If this hypothesis is correct, the decrease in the instantaneous gradient during unloading may change in accordance with the degree of inhomogeneity, i.e., the inelastic deformation during unloading would be more pronounced when the sheet material has more random crystallographic orientations and would be decreased when the sheet material has a stronger basal texture. To confirm this, a loading-unloading simulation using sheets with random crystallographic orientations and a more pronounced texture was carried out. Figure 19 shows the initial (0001) pole figure of the simulation model with the more pronounced texture.

The stress-strain curves obtained by the loading-unloading simulation with the rolling texture (Fig. 3), the random orientations, and the more pronounced texture are shown in Fig. 20. Clearly, the sheet with random crystallographic orientations exhibits larger inelastic deformation during unloading than that with rolling texture, whereas inelastic deformation during unloading 


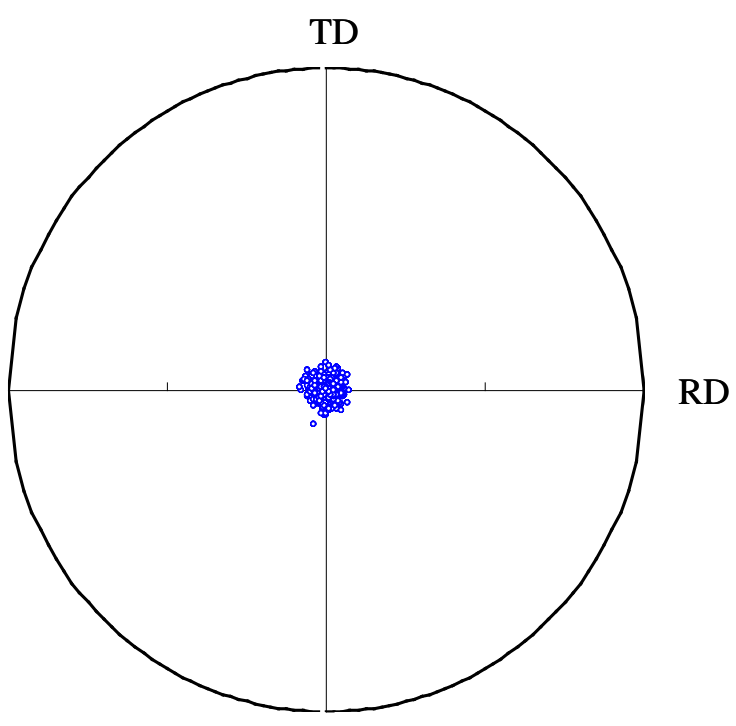

Fig. 19 (0001) pole figure used in the simulation model with a more pronounced texture.

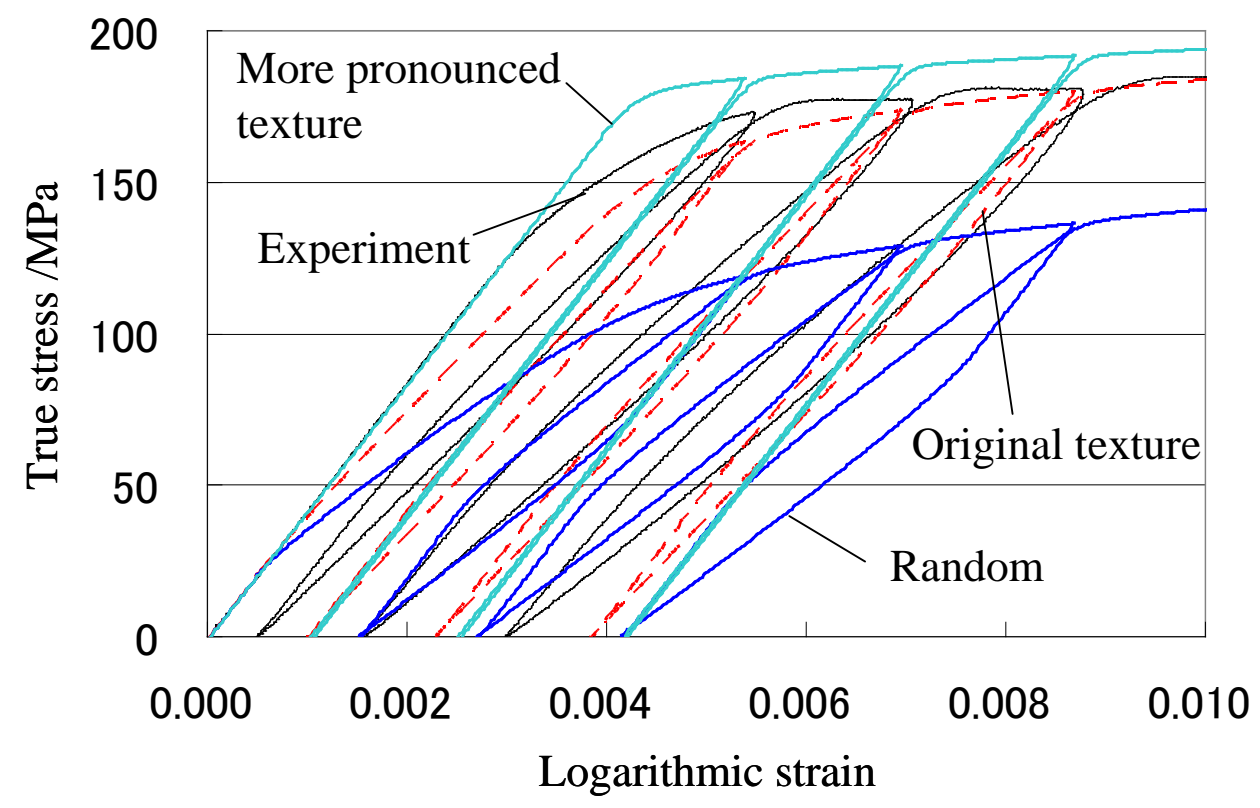

Fig. 20 Comparison of simulated stress-strain curve among three different initial crystallographic orientations. 
(a)

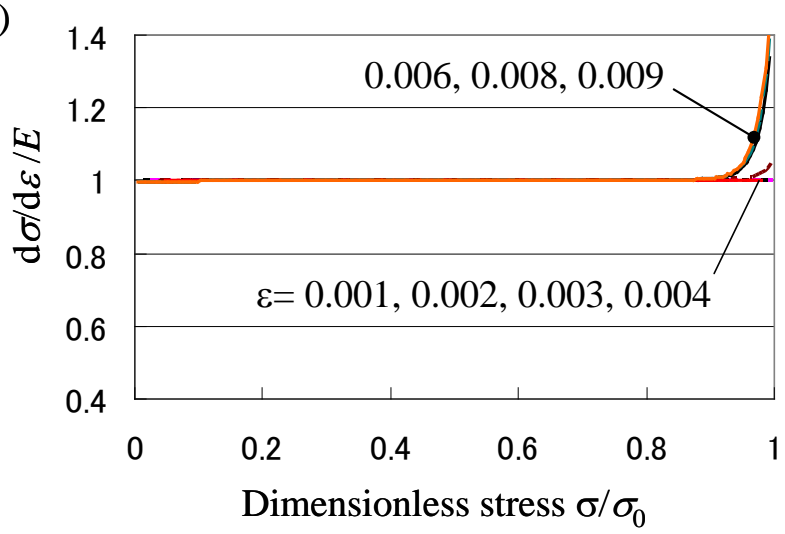

(b)

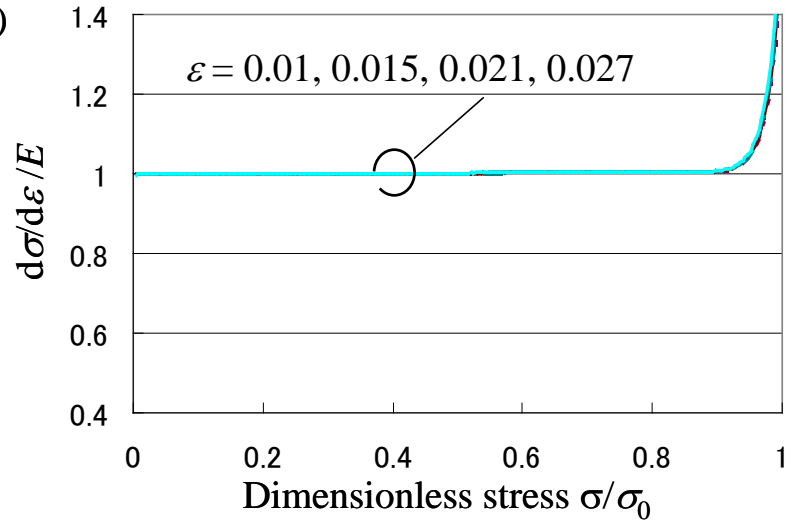

Fig. 21 Variation in simulated instantaneous gradient $\mathrm{d} \sigma / \mathrm{d} \varepsilon$ during unloading with a more pronounced texture in the unloaded strain range: (a) from 0.001 to 0.009 and (b) from 0.01 to 0.027 .

(a)

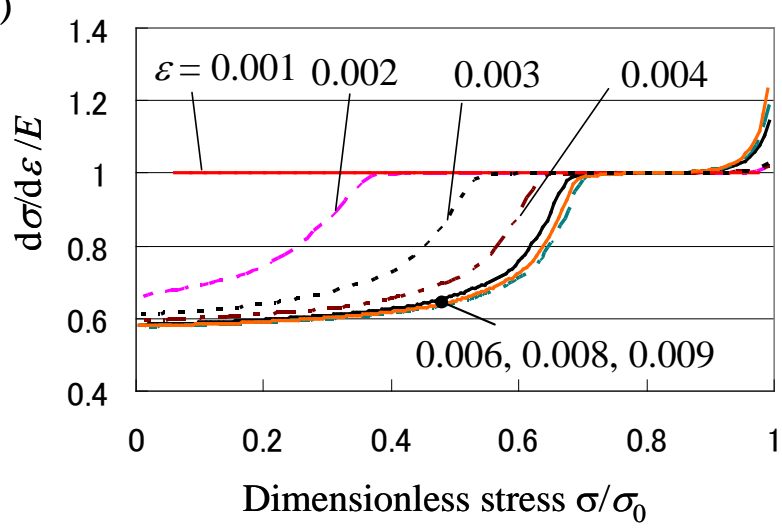

(b)

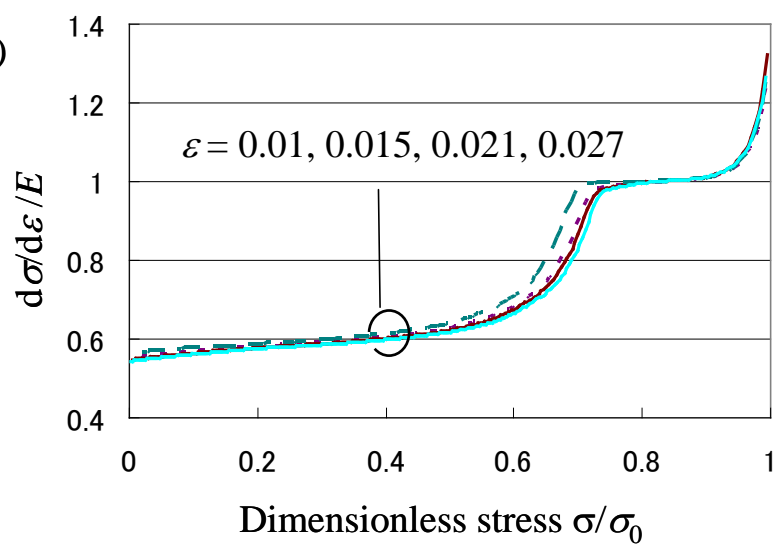

Fig. 22 Variation in simulated instantaneous gradient $\mathrm{d} \sigma / \mathrm{d} \varepsilon$ during unloading with random crystallographic orientations in the unloaded strain range: (a) from 0.001 to 0.009 and (b) from 0.01 to 0.027 .

is very small in the sheet with the more pronounced texture. Figures 21 and 22 show the variation of the instantaneous gradient $\mathrm{d} \sigma / \mathrm{d} \varepsilon$ with the stress obtained with the more pronounced texture and the random crystallographic orientations. For the random crystallographic orientations, the 
overall trend of the variation curve is similar to that for the rolling texture (Fig. 9). However, the decrease in the instantaneous gradient is much larger than that obtained with the rolling texture. On the other hand, in case of the more pronounced texture, inelastic deformation during
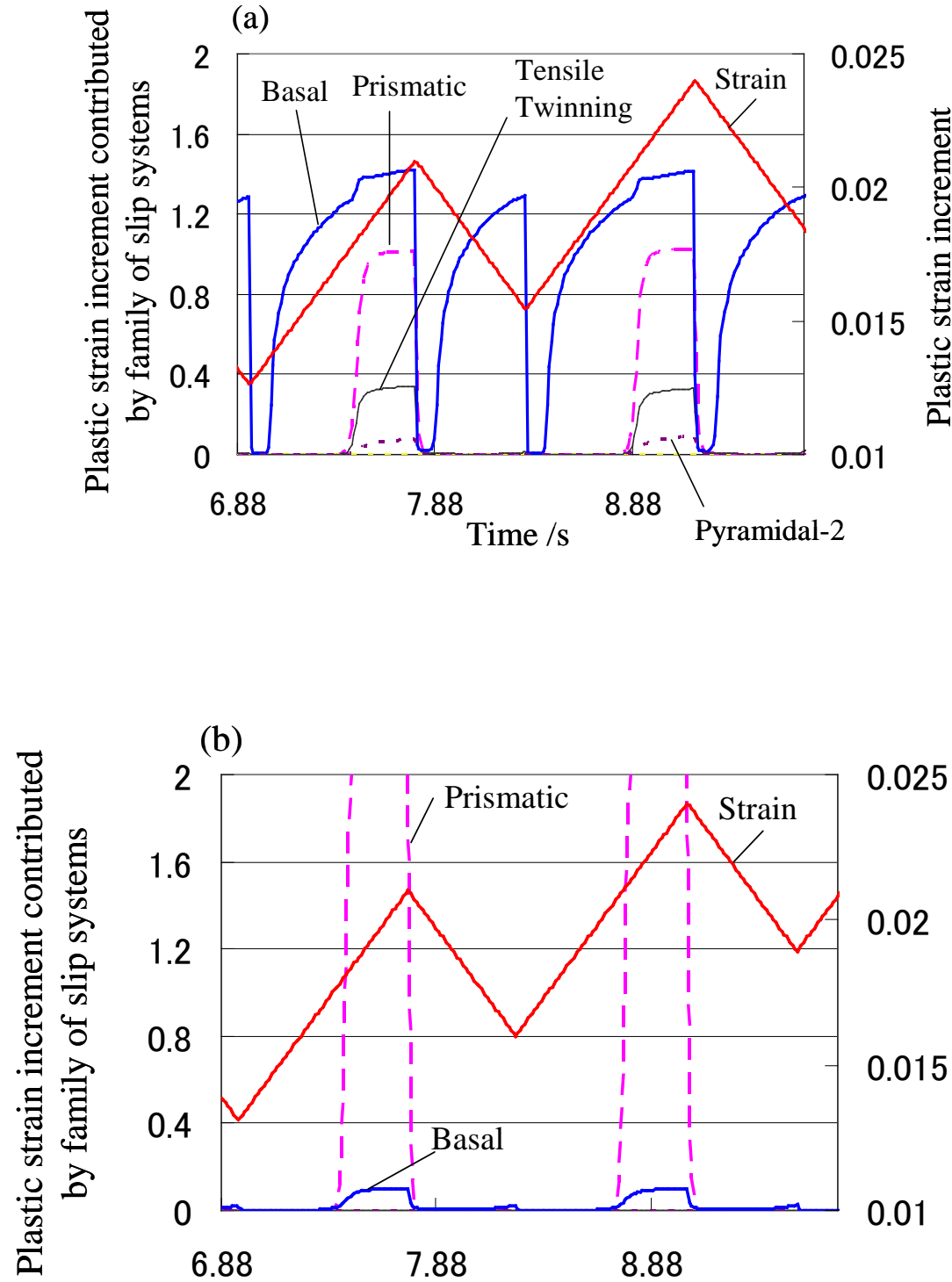

(b)

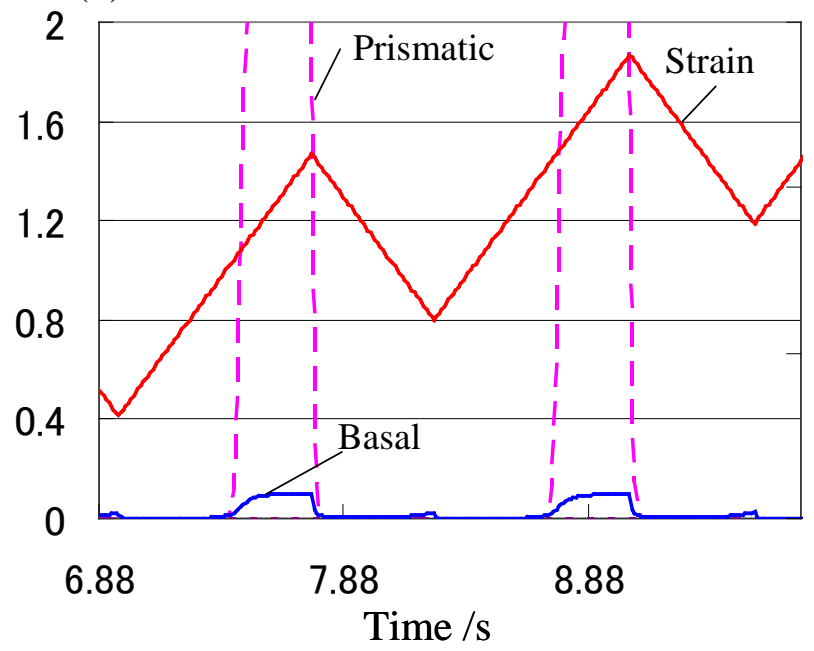

0.025

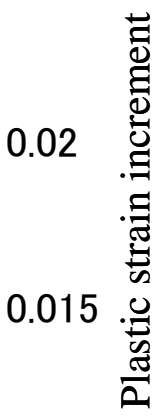

0.01

Fig. 23 Plastic strain increment contributed by family of slip systems during loadingunloading in the strain range from 0.012 to 0.024 . (a) Results with random crystallographic orientations, and (b) results with more pronounced texture. 
unloading is negligible. Figure 23 shows the simulated evolution of the plastic strain increment during the same period covered in Fig. 14. A tendency in the evolution of the plastic strain increment obtained with the random orientations is similar to that of the rolling texture (Fig. 14), but the magnitude of the plastic strain increment of the basal slip systems during unloading is larger than that of the rolling texture. On the other hand, the activation of the slip and twinning systems during unloading obtained with the more pronounced texture is very small. The above results clearly show that inelastic deformation during unloading observed in our results is attributable to the activation of the basal slip systems that is induced by the inhomogeneity of the material. Actually, the above tendency shown in Fig. 20 is also observed experimentally. Muránsky et al. (2009) reported that the inelastic behavior under uniaxial tension using an extruded Mg alloy is much less pronounced than that reported by Cáceres et al. (2003) using a cast $\mathrm{Mg}$ alloy. They concluded that this difference is induced by the fact that twinning is suppressed by the strong extrusion texture. Our simulation results clearly show that, besides tensile twinning, the activation of the basal slip systems is also one of the reasons for this difference.

\section{Conclusions}

In this study, the loading-unloading behavior of a Mg alloy sheet under uniaxial tension was simulated using the crystal-plasticity finite-element method. The mechanism of the inelastic behavior during unloading was examined in detail in terms of macroscopic and mesoscopic deformation, focusing on the effects of basal and nonbasal slip systems. The variation with stress of the instantaneous gradient during unloading was investigated for a macroscopic stress-strain 
curve, and the activity of each family of slip systems was examined for mesoscopic deformation. The following conclusions were drawn.

(1) Clear inelastic behavior arises during unloading in the simulation result, but the magnitude of the hysteresis is smaller than in the experiment. The variation with the stress of the instantaneous gradient obtained by the simulation is not in agreement with the experimental result. However, the trends that the instantaneous gradient decreases as the unloaded strain increases and that the variation curve saturates at an unloaded strain of about 0.01 are in good agreement.

(2) The slip and tensile twinning systems are activated during not only loading but also unloading. For instance, the prismatic slip systems are activated during only loading, whereas the basal slip systems are activated during both loading and unloading. On the other hand, the average sliding direction of the basal slip systems over all the grains during unloading is opposite to that during loading. The above tendencies also arise in each basal slip system in the grains. The mechanism of this behavior can be explained as follows. The overall stress level during loading is determined primarily by the prismatic slip systems because the activation of the prismatic slip systems is dominant during loading. Because the strength of the basal slip systems is much lower than that of the prismatic slip systems, they can be activated under the stress level during unloading. More precisely, the basal slip systems would be easily activated in the opposite direction when their Schmid's resolved shear stresses change sign during unloading because of the inhomogeneity of the material. These results indicate that one explanation for the inelastic behavior during unloading is that, because of the inhomogeneity of the material, the basal slip systems are easily activated owing to their low strength compared to that of the prismatic slip systems. 
(3) As expected from the above results, the activation of the basal slip systems and inelastic behavior during unloading change in accordance with the degree of inhomogeneity: the sheet with random orientations exhibits larger inelastic deformation and more pronounced activation of the basal slip systems during unloading than that with rolling texture, whereas inelastic deformation and activation of the basal slip systems during unloading is very small in the sheet with the more pronounced texture. These results show that inelastic deformation during unloading observed in our results is attributable to the activation of the basal slip systems that is induced by the inhomogeneity of the material.

The partial reversal of the tensile twinning systems may be an important factor in the inelastic behavior during unloading. However, to understand clearly the effect of basal and nonbasal slip on inelastic behavior we used a simple $\left\{\begin{array}{llll}1 & 0 & \overline{1} & 2\end{array}\right\}$ tensile twinning model in which untwinning is not taken into account. In future work, a more sophisticated tensile twinning model will be implemented and more elaborate studies of the inelastic behavior will be carried out.

It would also be interesting to use the crystal-plasticity finite-element method to carry out similar analyses of the inelastic behavior during unloading for BCC and FCC materials such as steel and aluminum alloys. This will also be future work.

\section{Acknowledgements}

The authors wish to acknowledge Professor M. Kuroda and Assistant Professor K. Yoshida of Yamagata University for their helpful advice on inelastic behavior. 


\section{REFERENCES}

Agnew, S.R., Tomé, C.N., Brown, D.W., Holden, T.M., Vogel, S.C., 2003. Study of slip mechanisms in a magnesium alloy by neutron diffraction and modeling. Scripta Materialia 48, 1003-1008.

Agnew S.R., Duygulu, Ö., 2005. Plastic anisotropy and the role of nonbasal slip in magnesium alloy AZ31B. International Journal of Plasticity 21, 1161-1193.

Asaro, R.J., Needleman, A., 1985. Texture development and strain hardening in rate dependent polycrystals. Acta Metallurgica 33, 923-953.

Bruni, C., Forcellese, A., Gabrielli, F., Simoncini, M., 2006. Air bending of AZ31 magnesium alloy in warm and hot forming conditions. Journal of Materials Processing Technology 177, 373-376.

Cáceres, C.H., Sumitomo, T., Veidt, M., 2003. Pseudoelastic behavior of cast magnesium AZ91 alloy under cyclic loading-unloading. Acta Materialia 51, 6211-6218.

Chen, F.K., Huang, T.B., 2003. Formability of stamping magnesium-alloy AZ31 sheets. Journal of Materials Processing Technology 142, 643-647.

Chen, F.K., Huang, T.B., Chang, C.K., 2003. Deep drawing of square cups with magnesium alloy AZ31 sheets. International Journal of Machine Tools \& Manufacture 43, 1553-1559.

Chino, Y., Furuta, T., Hakamada, M., Mabuchi, M., 2006a. Fatigue behavior of AZ31 magnesium alloy produced by solid-state recycling. Journal of Materials Research 41, 3229-3232.

Chino, Y., Lee, J.S., Sassa, K., Kamiya, A., Mabuchi, M., 2006b. Press formability of a rolled AZ31 Mg alloy sheet with controlled texture. Materials Letters 60, 173-176.

Chino, Y., Sassa, K., Kamiya, A., Mabuchi, M., 2008a. Stretch formability at elevated temperature of a crossrolled AZ31 Mg alloy sheet with different rolling routes. Materials Science and Engineering A 473, 195200.

Chino, Y., Kimura, K., Mabuchi, M., 2008b. Twinning behavior and deformation mechanisms of extruded AZ31 Mg alloy. Materials Science and Engineering A 486, 481-488. 
Choi, S.-H., Kim, D.H., Lee, H.W., Seong, B.S., Piao, K., Wagoner, R., 2009. Evolution of the deformation texture and yield locus shape in an AZ31 Mg alloy sheet under uniaxial loading. Materials Science and Engineering A 526, 38-49.

Clausen, B., Tomé, C.N., Brown, D.W., Agnew, S.R., 2008. Reorientation and stress relaxation due to twinning: Modeling and experimental characterization for Mg. Acta Materialia 56, 2456-2468.

Cleveland, R.M., Ghosh, A.K., 2002. Inelastic effects on springback in metals. International Journal of Plasticity 18, 769-785.

Diard, O., Leclercq, S., Rousselier, G., Cailletaud, G., 2005. Evaluation of finite element based analysis of 3D multicrystalline aggregates plasticity: Application to crystal plasticity model identification and the study of stress and strain fields near grain boundaries. International Journal of Plasticity 21, 691-722.

Doege, E., Droder, K., 2001. Sheet metal forming of magnesium wrought alloy - formability and process technology. Journal of Materials Processing Technology 115, 14-19.

Goo, E., Duerig, T., Melton, K., Sinclair, R., 1985. Mechanical twinning in Ti50Ni47Fe3 and Ti49Ni51 alloys. Acta Metallurgica 33, 1725-1733.

Graff, S., Brocks, W., Steglich, D., 2007. Yielding of magnesium: From single crystal to polycrystalline aggregates. International Journal of Plasticity 23, 1957-1978.

Hama, T., Nagata, T., Teodosiu, C., Makinouchi, A., Takuda, H., 2008. Finite-element simulation of springback in sheet metal forming using local interpolation for tool surfaces. International Journal of Mechanical Sciences 50, 175-192.

Hama, T., Kariyazaki, Y., Ochi, K., Fujimoto, H., Takuda, H., 2010a. Springback characteristics of Magnesium alloy AZ31B in draw-bending. Materials Transactions 51(4), 685-693.

Hama, T., Fujimoto, H., Takuda, H., 2010b. Crystal Plasticity finite element analysis of loading-unloading behavior in magnesium alloy sheet. Proc. NUMIFORM ‘10, Pohang, Korea, June, 2010, 802-809, CDROM.

Harder, J., 1999. A crystallographic model for the study of local deformation processes in polycrystals. International Journal of Plasticity 15, 605-624. 
Jones, E.R.W., Munro, W., 1953. The "elastic hysteresis” of uranium. Journal of the Mechanics and Physics of Solids 1, 182-188.

Kaneko, J. Sugamata, M., 2004. Mechanical properties and formability of magnesium alloy sheets. Journal of Japan Institute of Light Metals 54(11), 484-492 (in Japanese).

Kawka, M., Makinouchi, A., 1995. Shell-element formulation in the static explicit FEM code for the simulation of sheet stamping. Journal of Materials Processing Technology 50, 105-115.

Kim, H.J., Choi, S.C., Lee, K.T., Kim, H.Y., 2008. Experimental determination of forming limit diagram and springback characteristics of AZ31B Mg alloy sheets at elevated temperatures. Materials Transactions 49(5), 1112-1119.

Koike, J., 2005. Enhanced deformation mechanisms by anisotropic plasticity in polycrystalline Mg alloys at room temperature. Metallurgical and Materials Transactions A 36, 1689-1696.

Koike, J., 2009. Deformation mechanisms of magnesium alloys at ambient temperature. J. JILM. 59, 272-276 (in Japanese).

Kuwabara, T., Kumano, Y., Ziegelheim, J., Kurosaki, I., 2009. Tension-compression asymmetry of phosphor bronze for electronic parts and its effect on bending behavior. International Journal of Plasticity 25, 17591776.

Lebensohn, R.A., Tomé, C.N., 1993. A self-consistent anisotropic approach for the simulation of plastic deformation and texture development of polycrystals: Application to zirconium alloys. Acta Metallurgica et Materialia 41, 2611-2624.

Lee, S., Chen, Y.H., Wang, J.Y., 2002. Isothermal sheet formability of magnesium alloy AZ31 and AZ61. Journal of Materials Processing Technology 124, 19-24.

Lee, Y.S., Kim, M.C., Kim, S.W., Kwon, Y.N., Choi, S.W., Lee, J.H., 2007. Experimental and analytical studies for forming limit of AZ31 alloy on warm sheet metal forming. Journal of Materials Processing Technology 187-188, 103-107.

Lévesque, J., Inal, K., Neale, K.W., Mishra, R.K., 2010. Numerical modeling of formability of extruded magnesium alloy tubes. International Journal of Plasticity 26, 65-83. 
Li, Y., Enoki, M., 2008. Recovery behavior of pure magnesium in cyclic compression-quick unloadingrecovery process at room temperature investigated by AE. Materials Transactions 49, 1800-1805.

Lou, X.Y., Li, M., Boger, R.K., Agnew, S.R., Wagoner, R.H., 2007. Hardening evolution of AZ31B Mg sheet. International Journal of Plasticity 23, 44-86.

Luo, L., Ghosh, A. K., 2003. Elastic and inelastic recovery after plastic deformation of DQSK steel sheet. Journal of Engineering Materials and Technology 125, 237-246.

Mann, G.E., Sumitomo, T., Cáceres, C.H., Griffiths, J.R., 2007. Reversible plastic strain during cyclic loadingunloading of Mg and Mg-Zn alloys. Materials Science and Engineering A 456, 138-146.

Mayama, T., Aizawa, K., Tadano, Y., Kuroda, M., 2009. Influence of twinning deformation and lattice rotation on strength differential effect in polycrystalline pure magnesium with rolling texture. Computational Materials Science 47, 448-455.

Mordike, B.L., Ebert, T., 2001. Magnesium properties - applications - potential. Materials Science and Engineering A 302, 37-45.

Morestin, F., Boivin, M., 1996. On the necessity of taking into account the variation in the Young modulus with plastic strain in elastic-plastic software. Nuclear Engineering and Design 162, 107-116.

Muránsky, O., Carr, D.G., Sittner, P., Oliver, E.C., 2009. In situ neutron diffraction investigation of deformation twinning and pseudoelastic-like behavior of extruded AZ31 magnesium alloy. International Journal of Plasticity 25, 1107-1127.

Nakamachi, E., Tam, N.N., Morimoto, H., 2007. Multi-scale finite element analyses of sheet metals by using SEM-EBSD measured crystallographic RVE models. International Journal of Plasticity 23, 450-489.

Pérez, R., Benito, J.A., Prado, J.M., 2005. Study of the inelastic response of TRIP steels after plastic deformation. ISIJ International, 45, 1925-1933.

Pierce, D., Asaro, R.J., Needleman, A., 1983. Material rate dependence and localized deformation in crystalline solids. Acta Metallurgica 31, 1951-1976.

Prakash, A., Weygand, S.M., Riedel, H., 2009. Modeling the evolution of texture and grain shape in Mg alloy AZ31 using the crystal plasticity finite element method. Computational Materials Science 45, 744-750. 
Proust, G., Tomé, C.N., Jain, A., Agnew, S.R., 2009. Modeling the effect of twinning and detwinning during strain-path changes of magnesium alloy AZ31. International Journal of Plasticity 25, 861-880.

Reed-Hill, R.E., Robertson, W.D., 1958. Pyramidal slip in magnesium, Transactions of Metallurgical Society of AIME 212, 256-259.

Reed-Hill, R.E., Dahlberg, E.P., Slippy, Jr. W.A., 1965. Some anelastic effects in zirconium at room temperature resulting from prestrain at $77 \mathrm{~K}$. Transactions of the Metallurgical Society of AIME 233, 17661770.

Salem, A.A., Kalidindi, S.R., Semiatin, S.L., 2005. Strain hardening due to deformation twinning in $\alpha$ titanium: Constitutive relations and crystal-plasticity modeling. Acta Materialia 53, 3495-3502.

Staroselsky, A., Anand, L., 2003. A constitutive model for hcp materials deforming by slip and twinning: Application to magnesium alloy AZ31B. International Journal of Plasticity 19, 1843-1864.

Styczynski, A., Hartig, Ch., Bohlen, J., Letzig, D., 2004. Cold rolling textures in AZ31 wrought magnesium alloy. Scripta Materialia 50, 943-947.

Tadano, Y., 2010. Polycrystalline behavior analysis of pure magnesium by the homohenization method. International Journal of Mechanical Sciences 52 (2010), 257-265.

Tang, W., Zhang, S., Peng, Y., Li, D., 2009. Simulation of magnesium alloy AZ31 sheet during cylindrical cup drawing with rate independent crystal plasticity finite element method. Computational Materials Science 46, 393-399.

Tomé, C.N., Lebensohn, R.A., Kocks, U.F., 1991. A model for texture development dominated by deformation twinning: Application to zirconium alloys. Acta Metallurgica et Materialia 39, 2667-2680.

Turner, P.A., Tomé, C.N., 1994. A study of residual stresses in zircaloy-2 with rod texture. Acta Metallurgica et Materialia 42, 4143-4153.

Walde, T., Riedel, H., 2007. Simulation of earing during deep drawing of magnesium alloy AZ31. Acta Materialia 55, 867-874.

Wu, X., Kalidindi, S.R., Necker, C., Salem, A.A., 2007. Prediction of crystallographic texture evolution and anisotropic stress-strain curves during large plastic strains in high purity $\alpha$-titanium using a Taylor-type crystal plasticity model. Acta Materialia 55, 423-432. 
Xie, C.L., Ghosh, S., Groeber, M., 2004. Modeling cyclic deformation of HSLA steels using crystal plasticity. Journal of Engineering Materials and Technology. Transactions of the ASME 126, 339-352.

Yoshida, F., Uemori, T., 2003. A model of large-strain cyclic plasticity and its application to springback simulation. International Journal of Mechanical Sciences 45, 1687-1702.

Yoshinaga, H., Horiuchi, R., 1964. On the nonbasal slip in magnesium crystals. Transactions of the Japan Institute of Metals 5, 14-21. 